\title{
An extremely hot white dwarf with a rapidly rotating K-type subgiant companion: UCAC2 46706450
}

\author{
Klaus Werner ${ }^{1}$, Nicole Reindl ${ }^{2}$, Lisa Löbling ${ }^{1}$, Ingrid Pelisoli ${ }^{2}$, Veronika Schaffenroth ${ }^{2}$, \\ Alberto Rebassa-Mansergas ${ }^{3,4}$, Puji Irawati ${ }^{5}$, and Juanjuan Ren ${ }^{6}$ \\ ${ }^{1}$ Institut für Astronomie und Astrophysik, Kepler Center for Astro and Particle Physics, Eberhard Karls Universität, Sand 1, 72076 \\ Tübingen, Germany \\ e-mail: werner@astro.uni-tuebingen.de \\ 2 Institut für Physik und Astronomie, Universität Potsdam, Karl-Liebknecht-Straße 24/25, Germany \\ ${ }^{3}$ Departament de Física, Universitat Politècnica de Catalunya, c/Esteve Terrades 5, 08860 Castelldefels, Spain \\ ${ }^{4}$ Institut d'Estudis Espacials de Catalunya, Ed. Nexus-201, c/Gran Capità 2-4, 08034 Barcelona, Spain \\ ${ }^{5}$ National Astronomical Research Institute of Thailand, Sirindhorn AstroPark, Donkaew, Mae Rim, Chiang Mai 50180, Thailand \\ ${ }^{6}$ Key Laboratory of Space Astronomy and Technology, National Astronomical Observatories, Chinese Academy of Sciences, Beijing \\ 100101, PR China
}

Received 3 June 2020 / Accepted 5 September 2020

\begin{abstract}
The subgiant UCAC2 46706450 is a late-type star with an ultraviolet (UV) excess. It was considered as a candidate to establish a sample of stars of spectral type F, G, and K with white dwarf (WD) companions that could be used to test binary evolution models. To verify the WD nature of the companion, UV spectroscopy has previously been performed by other authors. Via a detailed modelatmosphere analysis, we show that the UV source is an extremely hot WD with an effective temperature of $T_{\text {eff }}=105000 \pm 5000 \mathrm{~K}$, mass of $M / M_{\odot}=0.54 \pm 0.02$, radius of $R / R_{\odot}=0.040_{-0.004}^{+0.005}$, and luminosity of $L / L_{\odot}=176_{-49}^{+55}$, meaning that the compact object is just about to enter the WD cooling sequence. Investigating spectra of the cool star $\left(T_{\text {eff }}=4945 \pm 250 \mathrm{~K}\right)$, we found that it is a K-type subgiant with $M / M_{\odot}=0.8-2.4, R / R_{\odot}=5.9_{-0.5}^{+0.7}$, and $L / L_{\odot}=19_{-5}^{+5}$ that is rapidly rotating with $v \sin (i)=81 \mathrm{~km} \mathrm{~s}^{-1}$. Optical light curves reveal a period of two days and an $o$-band peak-to-peak amplitude of $0.06 \mathrm{mag}$. We suggest that it is caused by stellar rotation in connection with star spots. With the radius, we infer an extremely high rotational velocity of $v_{\text {rot }}=151_{-13}^{+18} \mathrm{~km} \mathrm{~s}^{-1}$, thus marking the star as one of the most rapidly rotating subgiants known. This explains chromospheric activity observed by $\mathrm{H} \alpha$ emission and emission-line cores in $\mathrm{Ca}$ II $\mathrm{H}$ and $\mathrm{K}$ as well as NUV flux excess. From equal and constant radial velocities of the WD and the K subgiant as well as from a fit to the spectral energy distribution, we infer that they form a physical, wide (though unresolved) binary system. Both components exhibit similar metal abundances and show iron-group elements with slightly oversolar (up to 0.6 dex) abundance, meaning that atomic diffusion in the WD atmosphere is not yet active due to a residual, weak radiation-driven wind. Kinematically and from its height above the Galactic plane, the system belongs to the Galactic thick disk, indicating that it is an old system and that the initial masses of both stars were close to $1 M_{\odot}$.
\end{abstract}

Key words. stars: individual: UCAC2 46706450 - stars: atmospheres - stars: abundances - stars: AGB and post-AGB white dwarfs - starspots

\section{Introduction}

Stellar multiplicity is an omnipresent outcome of the starformation process. About every other solar-type (mass $M \approx 0.7-$ $1.3 M_{\odot}$ ) star is found in the binary system (see Duchêne \& Kraus 2013 for a review). At solar metallicity, about one quarter of these stars is found in close binaries (orbital period $P<10^{4} \mathrm{~d}$, separation $a<10 \mathrm{AU}$ ), and the close-binary fraction is found to increase strongly with decreasing metallicity and may be as high as $53 \%$ at a metallicity of $[\mathrm{Fe} / \mathrm{H}]=-3$ dex (Moe et al. 2019). When the more massive member of the binary evolves off the main sequence, such close systems will eventually interact with each other by exchanging mass and angular momentum (Willems \& Kolb 2004). This in turn influences their evolution and can lead to a broad range of astrophysical phenomena that are absent for the life of single stars. Systems that will undergo a common-envelope event will end up as very close binaries with final orbital periods typically between 0.1 and $10 \mathrm{~d}$ (Nebot Gómez-Morán et al. 2011), or even merge (Han et al. 2002).
Systems that transferred mass via stable Roche-lobe overflow or wind accretion can be found at longer final periods of a few $10^{2}-10^{3} \mathrm{~d}$ (Pastetter \& Ritter 1989; Han et al. 2002; Nie et al. 2012; Chen et al. 2013). Evolved systems with periods longer than about $3000 \mathrm{~d}$ are thought to always remain detached (Van der Swaelmen et al. 2017).

Detailed studies of evolved binaries are fundamental for various reasons. The mass and period distribution of very short orbital period binaries provides important observational constraints on the poorly understood common-envelope phase (Ritter 1986; Schreiber et al. 2010; Davis et al. 2010; Zorotovic et al. 2011). In addition, these binaries can be employed to search for and study supernovae type Ia progenitor candidates (Napiwotzki et al. 2001; Geier et al. 2013; Santander-García et al. 2015; Rebassa-Mansergas et al. 2019; Reindl et al. 2020) or help us to understand how binary interactions alter the intrinsic properties of the stars (such as their atmospheric composition, rotational rates, pulsations, mass loss, dust formation, and circumstellar-envelope morphology; Van Winckel 2018). Last 
but not least, binaries that avoided mass exchange can be used to investigate the initial-final mass relation, which is a key constraint on stellar evolution theory and important to understanding the chemical enrichment and the efficiency of star formation in galaxies (Silvestri et al. 2005; Catalán et al. 2008; Zhao et al. 2012b; Baxter et al. 2014; Andrews et al. 2015).

Aiming to provide a large sample to test binary evolution models and type Ia supernovae formation channels, Parsons et al. (2016) established a group of 934 main-sequence FGK stars from the Large Sky Area Multi-Object Fiber Spectroscopic Telescope (LAMOST, Zhao et al. 2012a) survey and the Radial Velocity Experiment survey (Kordopatis et al. 2013), which show excess flux at ultraviolet (UV) wavelengths, and hence likely have a white dwarf (WD) companion. Such systems are still very rarely known in comparison to thousands of $\mathrm{M}$ stars with WD companions (Holberg et al. 2013; Rebassa-Mansergas et al. 2016). For nine objects in their sample, Parsons et al. (2016) obtained follow-up spectroscopy with the Hubble Space Telescope (HST).

One of these nine systems, UCAC2 46706450, is the subject of this work. A spectroscopic analysis of the late-type star was performed by various authors revealing, for example, an effective temperature of $T_{\text {eff }}=4905 \pm 16 \mathrm{~K}$ and a surface gravity of $\log g=2.90 \pm 0.04$ (Ho et al. 2017), indicating that the star is an early K subgiant. Assuming that the observed Ly $\alpha$ line in the HST spectrum is of photospheric origin, a model-atmosphere fit by Parsons et al. (2016) to the Ly $\alpha$ profile yielded $T_{\text {eff }}=$ $24000 \mathrm{~K}$ and a low surface gravity of $\log g \approx 5.0$, indicating that the hot component is a pre-WD object like a hot subdwarf.

In the present paper, we analyze in detail the UV spectrum of the compact companion and show that its temperature was strongly underestimated, and that it is among the hottest known WDs (Sect. 2). We then reassess the spectroscopic observations to characterize the K-type star (Sect. 3), search for radial velocity (RV) variations (Sect. 4), and investigate its Galactic population membership (Sect. 5). We derive stellar parameters of both components (Sect. 6) and examine the optical photometric variability (Sect. 7). We conclude in Sect. 8.

In the following, we refer to the spectroscopic binary components as UCAC2 46706450-A and UCAC2 46706450-B for the $\mathrm{K}$ subgiant and the hot WD, respectively.

\section{Analysis of UV spectroscopy of the hot white dwarf}

The star UCAC2 46706450 was observed on Oct. 30, 2014, with the Cosmic Origins Spectrograph (COS) aboard HST, using the G130M grating centered on $1291 \AA$ for a 2109 s exposure (dataset LCKY08010, PI: S. Parsons). The spectrum was retrieved from the MAST archive. The approximate useful wavelength coverage is 1138-1278 $\AA$ and 1296-1426 . The spectral resolution is about $0.1 \AA$. For our analysis, we smoothed the spectra with a $0.1 \AA$-wide boxcar to increase the signal-to-noise ratio (SNR). For comparison with observations, model spectra were convolved with a Gaussian $(F W H M=0.14 \AA)$. The spectrum and our model fit are shown in Fig. 1. We identified lines from highly ionized light metals, namely, C IV, N IV-V, O IV-V, Si IV$\mathrm{V}, \mathrm{S}$ VI as well as from iron-group elements, namely, Cr VI, Mn VI, Fe VI-VIII, and Ni VI. Evidently the spectrum is that of a very hot WD. Concerning the ionization stages observed in the UV, it appears similar to other hot objects, for example, the DA PG 0948+534 with $T_{\text {eff }}=105000 \mathrm{~K}$ (Werner et al. 2019) and the DAO central stars of Sh 2-216 and EGB 6 with $T_{\text {eff }}=95000 \mathrm{~K}$ and $105000 \mathrm{~K}$, respectively (Rauch et al. 2007; Werner et al. 2018b).

The broad Ly $\alpha$ profile is dominated by interstellar hydrogen, and the COS spectrum does not cover any helium line. Thus, the $\mathrm{H} / \mathrm{He}$ ratio is unknown, and in principle the object could be either a He-rich (DO or DOA) WD or a H-rich (DA or DAO) WD. Nevertheless, we show that the effective temperature and metal abundance measurements are rather independent of this.

The photospheric lines are blueshifted by $-10 \mathrm{~km} \mathrm{~s}^{-1}$. Accordingly, the observed spectrum is shifted to rest wavelengths in all figures presented here. Some of the most prominent interstellar lines were modeled. They are blueshifted between -15 and $-35 \mathrm{~km} \mathrm{~s}^{-1}$. From Ly $\alpha$, we derived a neutral $\mathrm{H}$ column density of $\log n_{H}=20.4 \pm 0.04$ toward the WD. Comparing the continuum shape of our final model and observation, we found a reddening of $E_{\mathrm{B}-\mathrm{V}}=0.03 \pm 0.01$. The models presented here are attenuated by this reddening value. We note the reddening derived by us also agrees with values reported in the $2 \mathrm{D}$ dust maps of Schlegel et al. (1998) and Schlafly \& Finkbeiner (2011), as well as with the lower limit provided by the 3D dust map of Lallement et al. (2018).

We used the Tübingen Model-Atmosphere Package $\left(\mathrm{TMAP}^{1}\right)$ to compute non-LTE, plane-parallel, line-blanketed atmosphere models in radiative and hydrostatic equilibrium (Werner \& Dreizler 1999; Werner et al. 2003, 2012). The models include $\mathrm{H}, \mathrm{He}, \mathrm{C}, \mathrm{N}, \mathrm{O}, \mathrm{Si}, \mathrm{P}, \mathrm{S}, \mathrm{Cr}, \mathrm{Mn}, \mathrm{Fe}, \mathrm{Co}$, and Ni. The employed model atoms are described in detail by Werner et al. (2018a). In addition, we performed line formation iterations (i.e., keeping the atmospheric structure fixed) for fluorine using the model atom presented in Werner et al. (2015).

\subsection{Effective temperature, surface gravity, and $\mathrm{H} / \mathrm{He}$ ratio}

We started our analysis by fixing the value of the surface gravity to $\log g=7.4$. At $T_{\text {eff }} \approx 100000 \mathrm{~K}$, this corresponds to a WD with a mass of $\approx 0.54 M_{\odot}$. We also fixed the $\mathrm{H} / \mathrm{He}$ ratio to the solar value. We then varied $T_{\text {eff }}$ and the metal abundances to obtain a good fit to the line features. We found $T_{\text {eff }}=105000 \pm 5000 \mathrm{~K}$ and the abundances listed in Table 1 . The abundance measurements are described in detail below (Sect. 2.2). Finally, we looked how $\log g$ can be constrained and how the $\mathrm{H} / \mathrm{He}$ ratio affects the derived atmospheric parameters.

The relatively small error in $T_{\text {eff }}$ follows from the fact that we can exploit several ionization balances, first of all, iron. We do not see Fe V, imposing a strict lower limit of $100000 \mathrm{~K}$. The relative strengths of Fe VI to Fe VII lines then yield the value of $105000 \mathrm{~K}$ (Fig. 2). In addition, at temperatures above $110000 \mathrm{~K}$, the only observed Fe VIII line (at 1148.2 ̊) becomes too strong (a problem with a blending Ni VI line is discussed in Sect. 2.2). Another sensitive indicator for $T_{\text {eff }}$ is oxygen. With increasing temperature, the O IV multiplets (Fig. 2) and O V $1371 \AA$ A become weaker, whereas the highly excited O V multiplet at $1420 \AA$ becomes stronger. The detection of N IV lines (around 1190 and $1271 \AA$ ) rules out a $T_{\text {eff }}$ value well over $105000 \mathrm{~K}$. The absence of the C III multiplet at $1175 \AA$, on the other hand, requires $T_{\text {eff }}$ above $100000 \mathrm{~K}$.

Next, we looked at how the surface gravity affects the model spectrum. Reducing $\log g$ from the fiducial value 7.4 to 7.1 results in unobserved sharp line cores of the C IV doublet at $1352 \AA$. An increase to $\log g=7.7$ results in too broad wings of the C IV lines

http://astro.uni-tuebingen.de/ TMAP 


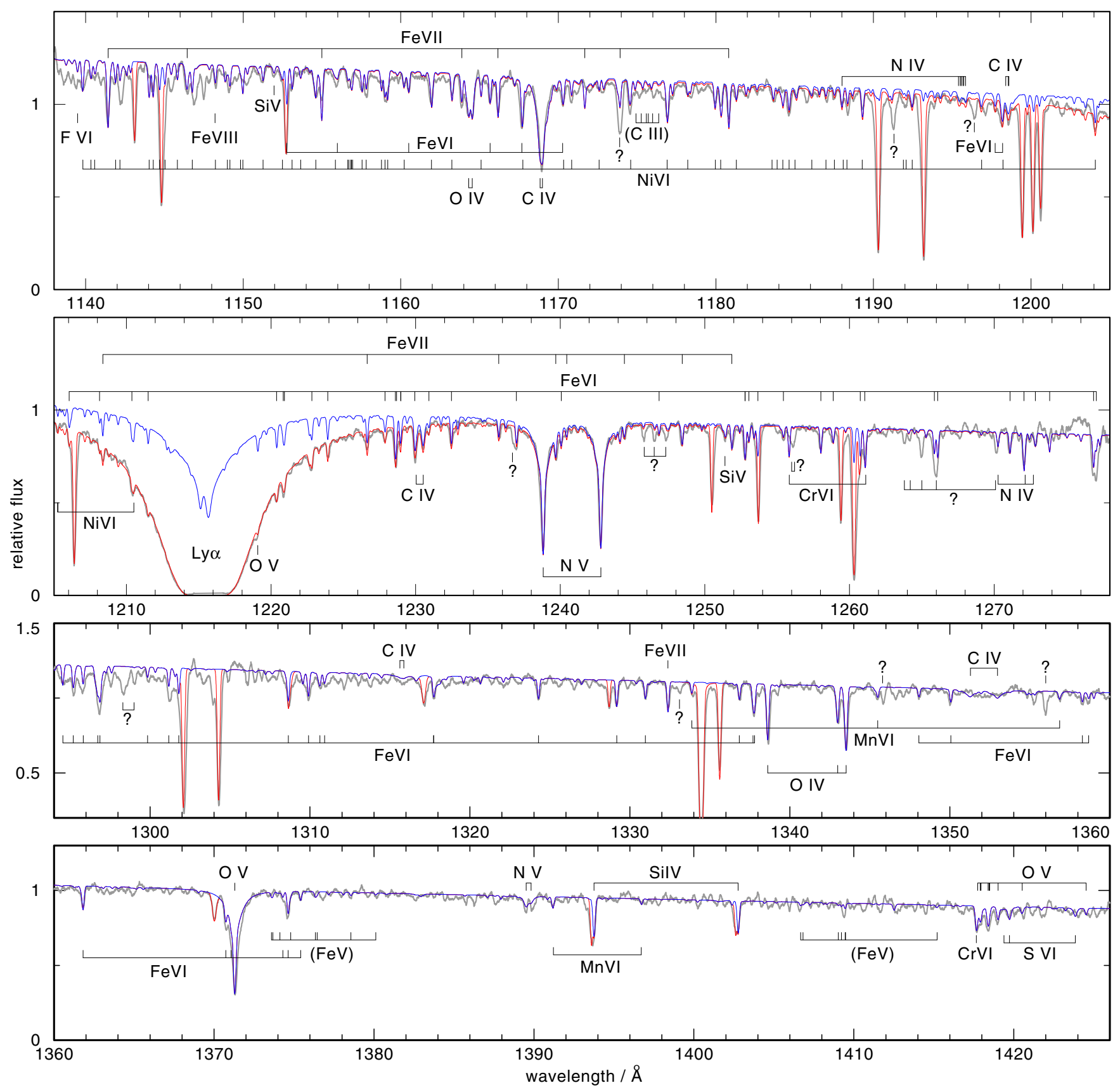

Fig. 1. HST/COS spectrum of the white dwarf in UCAC246706450 (gray) compared to a photospheric model spectrum (blue graph: $T_{\text {eff }}=105000 \mathrm{~K}, \log g=7.4$ ) with the measured metal abundances (Table 1) and assuming solar $\mathrm{H}$ and He abundances. The same model attenuated by interstellar lines is plotted in red. Prominent photospheric lines are identified. Identifications in brackets denote uncertain detections. Question marks indicate unidentified photospheric lines.

observed at 1178 and $1198 \AA$. We adopt $\log g=7.4 \pm 0.5$, with a conservative error estimate.

We then explored how the metal lines depend on different values of the $\mathrm{H} / \mathrm{He}$ ratio, which remains undetermined at the moment. The reduction of helium from the assumed solar value down to $1.3 \times 10^{-5}$ (mass fraction) is affecting the UV metal line strengths only marginally. The increase of helium to $98 \%$ is slightly shifting the ionization balance of metals to lower stages such that an increase of $T_{\text {eff }}$ by about $5000 \mathrm{~K}$ would be necessary to match the observed line strengths. Therefore, within error ranges, the measured metal abundances do not depend on the assumed $\mathrm{H} / \mathrm{He}$ ratio and hold irrespective of whether the star is a DA or a DO white dwarf.

\subsection{Metal abundances}

We present in detail how elemental abundances were inferred. The employed lines are identified in Fig. 1. The resulting abundance values are listed in Table 1.

\subsubsection{CNO}

Three C IV multiplets are detectable and were fit, namely $3 \mathrm{~d}-$ $4 \mathrm{f}, 3 \mathrm{~d}-4 \mathrm{p}$, and $3 \mathrm{p}-4 \mathrm{~s}$, at 1169,1198 , and $1230 \AA$, respectively. It is worthwhile to note that two other, more highly excited, C IV multiplets are not detectable: $4 \mathrm{p}-7 \mathrm{~d}$ at $1316 \AA$ and the blend of $4 \mathrm{~d}-7 \mathrm{f}$ and $4 \mathrm{f}-7 \mathrm{~g}$ at 1351 and $1353 \AA$. They serve to constrain the 

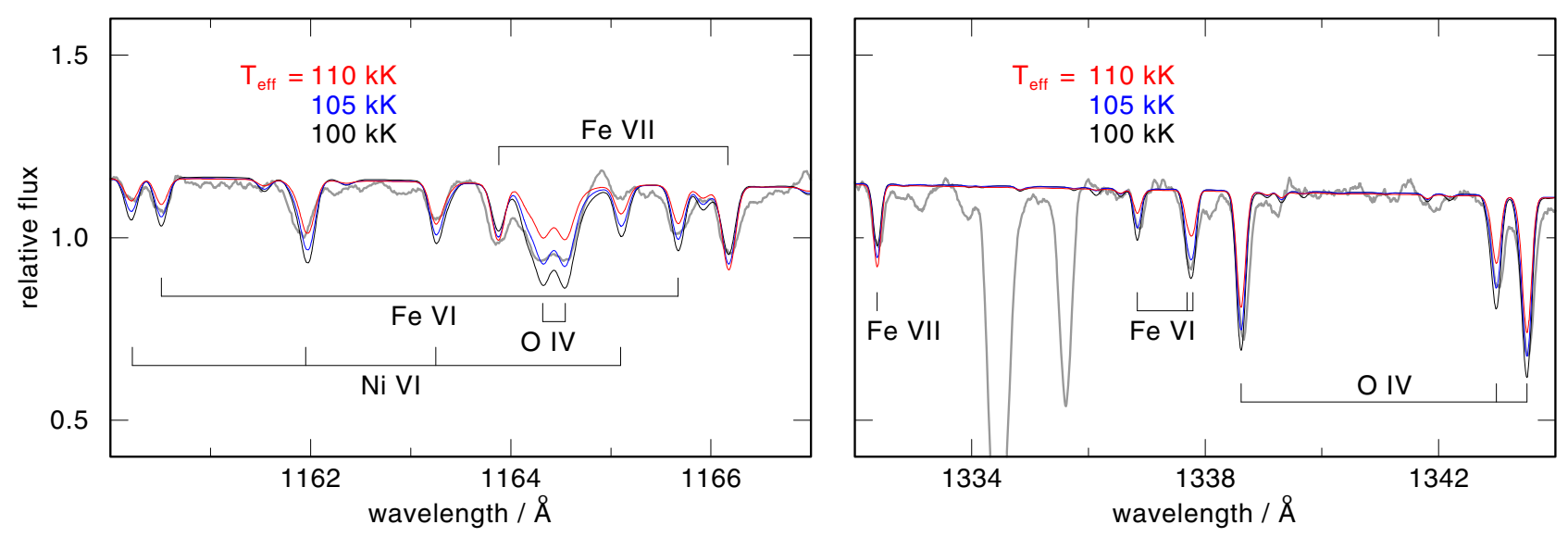

Fig. 2. Details of observed WD spectrum compared with three models with different temperatures $\left(T_{\text {eff }}=105000 \pm 5000 \mathrm{~K}\right)$ representing the error range in determination of $T_{\text {eff. }}$ Left: O IV multiplet gets weaker with increasing $T_{\text {eff }}$, as well as lines from Fe VI and Ni VI, while Fe VII lines get stronger. Right: same behavior of oxygen and iron lines in another wavelength range.

Table 1. Atmospheric parameters of the WD in UCAC2 46706450.

\begin{tabular}{rrr}
\hline \hline$T_{\text {eff }}(\mathrm{K})$ & $105000 \pm 5000$ & \\
$\log (g)\left(\mathrm{cm} \mathrm{s}^{-2}\right)$ & $7.4 \pm 0.5$ & \\
\hline Abundance & $X_{i}$ & {$\left[X_{i}\right]$} \\
\hline $\mathrm{C}$ & $2.0 \times 10^{-3}$ & -0.07 \\
$\mathrm{~N}$ & $2.0 \times 10^{-3}$ & 0.46 \\
$\mathrm{O}$ & $5.0 \times 10^{-3}$ & -0.06 \\
$\mathrm{~F}$ & $<5.0 \times 10^{-7}$ & $<0.16$ \\
$\mathrm{Si}$ & $8.0 \times 10^{-4}$ & 0.08 \\
$\mathrm{~S}$ & $3.1 \times 10^{-4}$ & 0.00 \\
$\mathrm{Cr}$ & $8.0 \times 10^{-5}$ & 0.70 \\
$\mathrm{Mn}$ & $3.0 \times 10^{-5}$ & 0.45 \\
$\mathrm{Fe}$ & $3.5 \times 10^{-3}$ & 0.46 \\
$\mathrm{Co}$ & $<3.0 \times 10^{-5}$ & $<0.91$ \\
$\mathrm{Ni}$ & $3.0 \times 10^{-4}$ & 0.64 \\
\hline
\end{tabular}

Notes. Abundances given in mass fractions (Col. 2) and logarithmic abundances relative to solar value (Col. 3; solar abundances from Asplund et al. 2009). Error limits for the abundances are \pm 0.5 dex.

upper limit on the $\mathrm{C}$ abundance and the lower limit on surface gravity (see Sect. 2.1).

Besides the strong $\mathrm{N} v$ resonance doublet, we observe a weak and highly excited N V multiplet $(4 s-5 p)$ at $1390 \AA$, and several $\mathrm{N}$ IV lines. There is a singlet at $1188 \AA\left({ }^{1} \mathrm{~S}-{ }^{1} \mathrm{P}^{\mathrm{o}}\right)$, which, however, is blended by a Ni VI line and a multiplet at $1196 \AA\left({ }^{3} \mathrm{D}-{ }^{3} \mathrm{P}^{\mathrm{o}}\right)$. A triplet appears at $1271 \AA\left({ }^{3} \mathrm{P}^{\mathrm{o}}-^{3} \mathrm{D}\right)$, but two components are blended by Fe VI lines.

Two prominent O IV multiplets are detected, namely a blend of components at $1164 \AA\left({ }^{2} \mathrm{D}-{ }^{2} \mathrm{~F}^{\mathrm{o}}\right)$ and the three components of the ${ }^{2} \mathrm{P}-{ }^{2} \mathrm{D}^{\mathrm{o}}$ transition at $1338.6 \AA, 1343.0 \AA$, and $1343.5 \AA$. We also detect several O V lines: the prominent O V $1371 \AA$, the ${ }^{1} \mathrm{D}-{ }^{1} \mathrm{D}^{\mathrm{o}}$ singlet at $1219 \AA$, and an accumulation of weak lines in the region $1418-1426 \AA$, which stem from the transitions ${ }^{3} \mathrm{P}^{\mathrm{o}}-{ }^{3} \mathrm{D}$ and ${ }^{3} \mathrm{D}-{ }^{3} \mathrm{~F}^{\mathrm{o}}$.

\subsubsection{Light metals: $\mathrm{F}, \mathrm{Si}$, and $\mathrm{S}$}

We detect a very weak feature at the position of the F VI $1139.5 \AA$ line, which is often observed in hot WDs (e.g., EGB 6, Werner

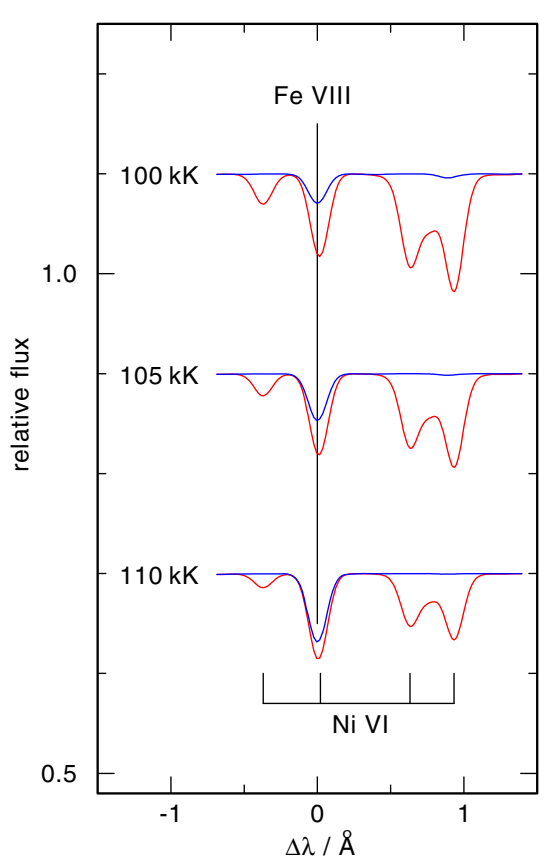

Fig. 3. Effect of increasing temperature on the $\mathrm{Ni} \mathrm{VI/Fe} \mathrm{VIII} \mathrm{line} \mathrm{blend}$ at $1148.2 \AA\left(T_{\text {eff }}=100,105\right.$, and $110 \mathrm{kK}$, from top to bottom). Blue graphs show spectra computed without Ni lines, that is to say, we only see the Fe VIII line profile. The nickel lines become weaker and the iron line becomes stronger. As a result, the line $\mathrm{Fe}+\mathrm{Ni}$ blend stays almost constant. The models have abundances of $\mathrm{Fe}=3.5 \times 10^{-3}$ and $\mathrm{Ni}=3.0 \times 10^{-4}$ by mass.

et al. 2018b). It can be fit with a solar fluorine abundance, however, this result is regarded as uncertain given the poor SNR at the blue edge of the COS spectrum. We therefore adopted the solar abundance as an upper limit.

Besides the SiIV resonance doublet at $1394 / 1402 \AA$, we detect two weak Si V lines at 1152.0 and $1251.4 \AA$, which are components of the ${ }^{3} \mathrm{P}^{\mathrm{o}}-{ }^{3} \mathrm{P}$ and ${ }^{3} \mathrm{P}^{\mathrm{o}}-{ }^{3} \mathrm{D}$ transitions, respectively. The Si IV doublet is blended by an ISM component that is blueshifted by $-25 \mathrm{~km} \mathrm{~s}^{-1}$ relative to the photospheric one. A weak S VI line at $1419.7 \AA$ is identified, which is the strongest of three components of the $4 d-5 p$ transition, allowing for a sulfur abundance measurement. 

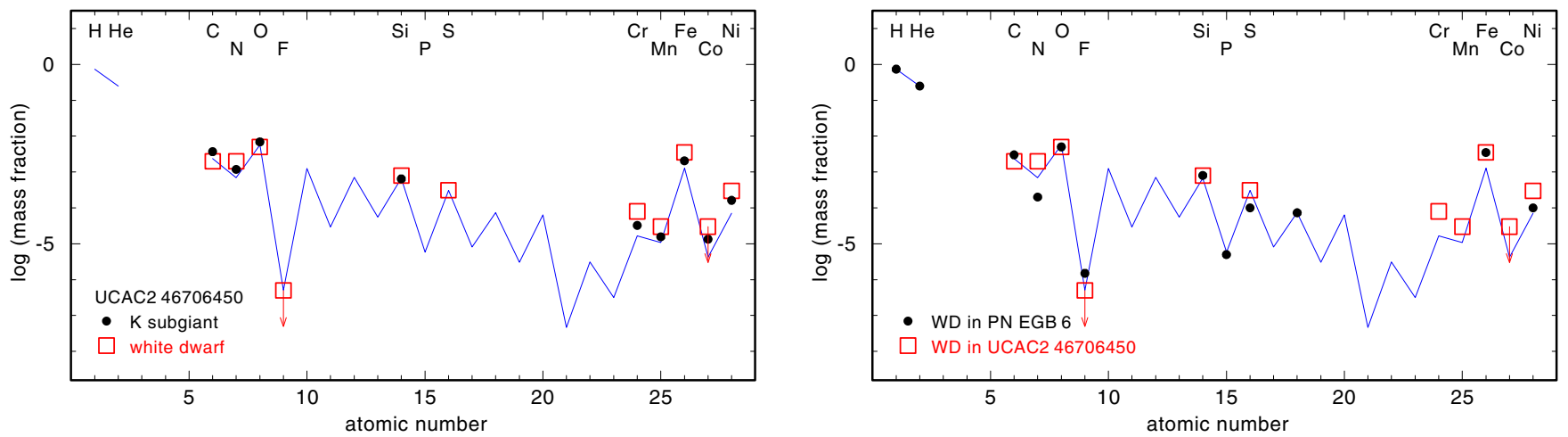

Fig. 4. Left: element abundances measured in the WD (red squares) and in the K subgiant (black dots) of the binary UCAC2 46706450. Right: abundances in the WD of UCAC2 46706450 (red squares), compared to the DAO WD in EGB 6 (black dots). The blue line indicates solar abundances.

\subsubsection{Iron group elements: $\mathrm{Cr}, \mathrm{Mn}, \mathrm{Fe}, \mathrm{Co}$, and $\mathrm{Ni}$}

A line of Cr VI is visible at $1417.7 \AA$. Another one, at $1261.1 \AA$, is a blend with an Fe VI line. A third one is seen at $1255.8 \AA$, but this is blended with a broad, unidentified feature. Five weak Mn VI lines are detected at 1333.9, 1345.5, 1356.9, 1391.2, and $1396.7 \AA$

As mentioned previously, we identified iron lines from Fe VIVIII. Care must be taken when using the only Fe VIII line (at $1148.2 \AA$ ) as a temperature indicator (Fig. 3). Its strength increases with $T_{\text {eff }}$, but there is a blending Ni VI line whose

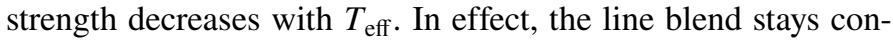
stant in the temperature regime discussed here. We do not see lines of $\mathrm{FeV}$. Some of the $\mathrm{Fe} \mathrm{V}$ lines that we see in models at slightly lower temperature in the wavelength region of 1373-1414 $\AA$ are indicated in Fig. 1 .

Our models predict many Co VI lines, mainly in the shortwavelength range of the COS spectrum, provided the cobalt abundance is high enough. We do not detect any such line and derive an upper abundance limit.

Many Ni VI lines are found, mainly in the short wavelength region up to about $1210 \AA$. Similarly to iron, Ni v lines are absent due to the high effective temperature.

\subsubsection{Unidentified lines}

A number of photospheric lines remain unidentified. The strongest appear at these wavelengths: 1173.6, 1191.0, 1195.7, 1236.4, 1246.2, 1256.3, 1266.9, 1298.3, 1332.8, 1345.5, and $1355.7 \AA$. These are indicated in Fig. 1 with a question mark. We also found these lines in other hot WDs or central stars of planetary nebulae (PNe) and suspect that some of them could be unknown Fe VII lines.

\subsubsection{Summary of abundance analysis of the WD}

Within error limits, the abundances of light metals metals are solar (Table 1, Fig. 4). For the iron-group elements a slight enhancement (0.45-0.70 dex oversolar) is found. This overabundance might point to radiative acceleration acting in the atmosphere, but we report below that the K subgiant also exhibits an overabundance of these elements (left panel of Fig. 4). Hence, the element abundance pattern probably reflects the initial metallicity of both stars. In the right panel of Fig. 4, we compare the abundances found for UCAC2 46706450-B with those of the WD central star of the PN EGB 6, which has the same $T_{\text {eff }}$ and $\log g$ (Werner et al. 2018b). Apart from nitrogen, the abundance patterns are rather similar.

\section{Optical and IR spectroscopy of the K star}

Subgiant UCAC2 46706450-A dominates the flux in the optical and infrared (IR) wavelength range. Optical spectroscopy $(\lambda \approx$ 3700-9000 $)$ ) of the system was obtained in course of the LAMOST survey (resolving power $R \approx 1800)$. Furthermore, moderately high-resolution $(R \approx 22500)$ IR $(\lambda \approx 15100-17000 \AA)$ spectroscopy was obtained with the Apache Point Observatory Galactic Evolution Experiment (APOGEE; Majewski et al. 2017). In the course of the Gaia mission, a medium-resolution $(R \approx 11700)$ spectrum over the wavelength range $8450-8720 \AA$ (around the Ca II triplet) was collected with the Radial Velocity Spectrometer.

Additional follow-up spectroscopy were obtained at the 5.1-m Palomar Hale telescope in California, USA, and the Thai National Telescope (TNT) at the National Observatory in Thailand. One spectrum was obtained at the Palomar Hale Telescope during the night of the Jan. 20, 2014. We used the double spectrograph together with the 1200 lines/mm grating in the red and the 600 lines $/ \mathrm{mm}$ grating in the blue. The observations were carried out using the long slit with $1^{\prime \prime}$ width. This configuration resulted in a wavelength coverage of $\approx 7600-9300 \AA$ in the red and $\approx 3500-6500 \AA$ in the blue at $R=6400$ and $R=1400$, respectively. The spectrum was reduced and calibrated using the pamela (Marsh 1989) and molly ${ }^{2}$ softwares, respectively. Thirteen additional spectra were obtained during the 2015-2017 period at the TNT using the Middle Resolution fiber-fed Echelle Spectrograph (MRES). The spectra covered the $\approx 4300-8800 \AA$ wavelength range at $R=15000$. The spectra were reduced and calibrated using the DECH software package.

The two LAMOST spectra from data release 2 (DR2) were analyzed by Luo et al. (2016), who derived $T_{\text {eff }}, \log g,[\mathrm{Fe} / \mathrm{H}]$, as well as the RV values from the individual observations. These spectra were also analyzed by Ho et al. (2017), who provide results for $T_{\text {eff }}, \log g,[\mathrm{Fe} / \mathrm{H}]$, as well as the $\alpha$-enhancement $[\alpha / \mathrm{M}]$. Two additional LAMOST spectra were obtained within the DR5 that were analyzed (along with the previous spectra) by Luo et al. (2019). Abundances of several elements (C, Cl, N, O, $\mathrm{Mg}, \mathrm{Al}, \mathrm{Si}, \mathrm{P}, \mathrm{K}, \mathrm{Ca}, \mathrm{Ti}, \mathrm{V}, \mathrm{Cr}, \mathrm{Mn}, \mathrm{Fe}, \mathrm{Co}, \mathrm{Ni}$, and Rb), $T_{\mathrm{eff}}$,

2 Tom Marsh's molly package is available at http://deneb.astro. warwick.ac.uk/phsaap/software 
Table 2. Atmospheric parameters, masses as obtained from evolutionary calculations, and radii and luminosities of the subgiant UCAC2 46706450-A and the white dwarf UCAC2 46706450-B.

\begin{tabular}{lll}
\hline \hline & $\mathrm{UCAC} 246706450-\mathrm{A}$ & $\mathrm{UCAC} 246706450-\mathrm{B}$ \\
\hline$T_{\text {eff }}(\mathrm{K})$ & $4945 \pm 250$ & $105000 \pm 5000$ \\
$\log g\left(\mathrm{~cm} \mathrm{~s}^{-2}\right)$ & $3.04 \pm 0.25$ & $7.4 \pm 0.5$ \\
$M / M_{\odot}$ & $0.8-2.4$ & $0.54 \pm 0.02$ \\
$R / R_{\odot}$ & $5.9_{-0.5}^{+0.7}$ & $0.040_{-0.004}^{+0.005}$ \\
$L / L_{\odot}$ & $19_{-5}^{+5}$ & $176_{-49}^{+55}$ \\
\hline
\end{tabular}

$\log g$, and RV from the APOGEE spectrum are provided by the APOGEE Stellar Parameter and Chemical Abundances Pipeline (ASPCAP, García Pérez et al. 2016). Some of these element abundances relevant for the comparison with the WD companion are depicted in the left panel of Fig. 4. The iron-group elements are slightly enhanced, by $0.2-0.5 \mathrm{dex}$. An interesting point to notice is that ASPCAP predicts an unusually high rotational velocity of $v \sin (i)=80.76 \mathrm{~km} \mathrm{~s}^{-1}$. Atmospheric parameters from the APOGEE spectrum were also derived with the data driven method called the Cannon (Ness et al. 2015; Casey et al. 2016). However, the $\chi^{2}$ of the Cannon fit is quite large, indicating that the parameters and abundances are not reliable, and thus we did not consider these values further. Finally, the Gaia DR2 also provides values for $T_{\text {eff }}$ and RV.

In Table 2, we provide the average of the $T_{\text {eff }}$ and $\log g$ values derived by the various analyses and give an estimate of the error, which also includes the systematic error (estimated from the scatter of the results from the individual analyses). We note that the fits to the optical spectrum do not consider the contribution of the flux of the WD to the blue part of the spectrum. On the other hand, values from the fit to the LAMOST spectrum do not differ too much from what is obtained from the APOGEE and Gaia spectra.

A closer inspection of the LAMOST spectra reveals that the line cores of the $\mathrm{Ca}$ II $\mathrm{H}$ and $\mathrm{K}$ doublet (Fig. 5) appear in emission. The double-peaked $\mathrm{H} \alpha$ emission (also seen in the TNT spectra, Fig. 6), can be explained by the superposition of a very broad emission line plus a photospheric absorption line. The strength of these emission lines is found to be time variable. Emissions in these lines are typical for chromospherically active stars (Wilson 1963, 1968; Gray \& Corbally 2009).

\section{Radial velocity variability}

The scatter of the RV values from the three individual APOGEE spectra (which were all obtained within one month) is smaller than $1 \mathrm{~km} \mathrm{~s}^{-1}$, which already indicates that the system is very likely not a close binary. We also searched for RV variations using the values we measured from the LAMOST, Palomar, and TNT spectra, as well as those provided in the catalogs for the APOGEE and Gaia spectra (total time coverage is about four years, see Table 3). In order to check if the RV variations are significant or are merely produced by random fluctuations, we followed the approach as outlined in Maxted et al. (2001) and Geier et al. (2015). For this, we calculated the inverse-variance weighted mean velocity from all RV measurements. Assuming this mean velocity to be constant, we calculated the $\chi^{2}$. Comparing this value with the $\chi^{2}$ distribution for the appropriate number of degrees of freedom, the probability $p$ of obtaining

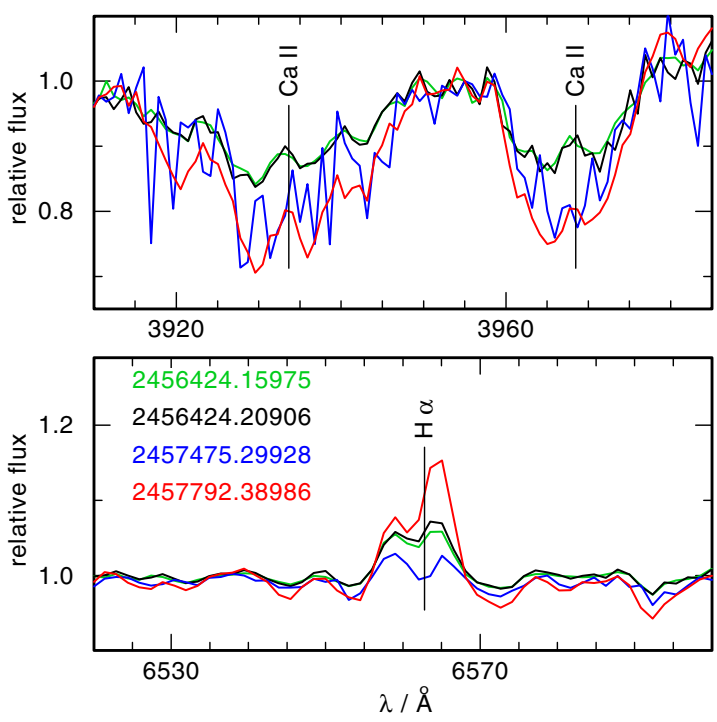

Fig. 5. Time-variable emission line cores in the $\mathrm{Ca}$ II $\mathrm{H}$ and $\mathrm{K}$ doublet and $\mathrm{H} \alpha$ emission observed in the LAMOST spectra indicate that UCAC2 46706450-A is chromospherically active. The HJD of the observations is provided.

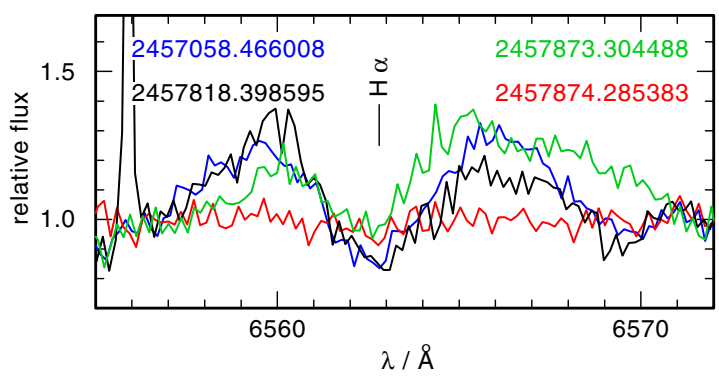

Fig. 6. Time-variable $\mathrm{H} \alpha$ emission observed in TNT spectra indicate that UCAC2 46706450-A is chromospherically active. The HJD of the observations is provided.

Table 3. HJD and radial velocities of UCAC2 46706450-A as measured from the different spectra.

\begin{tabular}{cll}
\hline \hline \multicolumn{1}{c}{ HJD } & $v_{\text {rad }}\left(\mathrm{km} \mathrm{s}^{-1}\right)$ & survey \\
\hline 2456424.148720 & $-22.0 \pm 10.6$ & LAMOST \\
2456424.160200 & $-23.7 \pm 10.6$ & LAMOST \\
2456424.171710 & $-21.6 \pm 10.6$ & LAMOST \\
2456424.190910 & $-22.9 \pm 10.5$ & LAMOST \\
2456424.210070 & $-20.3 \pm 10.5$ & LAMOST \\
2456424.228520 & $-18.6 \pm 10.6$ & LAMOST \\
2456678.061937 & $-21.9 \pm 6.0$ & Palomar \\
2456709.957320 & $-18.81318 \pm 0.15$ & APOGEE \\
2456717.996280 & $-17.94056 \pm 0.12$ & APOGEE \\
2456735.937290 & $-19.22651 \pm 0.17$ & APOGEE \\
2457058.466008 & $-27.2 \pm 3.4$ & TNT \\
2457475.290870 & $-26.0 \pm 10.4$ & LAMOST \\
2457475.300180 & $-26.0 \pm 10.4$ & LAMOST \\
2457475.309490 & $-21.5 \pm 10.5$ & LAMOST \\
2457792.389120 & $-31.5 \pm 13.2$ & LAMOST \\
2457817.394727 & $-33.1 \pm 3.4$ & TNT \\
2457818.398595 & $-21.0 \pm 3.4$ & TNT \\
2457819.380124 & $-18.1 \pm 4.1$ & TNT \\
2457819.400669 & $-30.3 \pm 7.3$ & TNT \\
\hline
\end{tabular}


the observed value of $\chi^{2}$ or higher from random fluctuations around a constant value can be calculated. We obtain a value of $\log p=-1.8$, and hence conclude that UCAC2 46706450-A is not significantly RV variable and very likely not a close binary. ${ }^{3}$

It is worth mentioning that Rebassa-Mansergas et al. (2017) claimed UCAC2 46706450-A to be RV variable. They obtained the RV values by fitting the $\mathrm{Na}$ I absorption doublet at around $5900 \AA$ A. However, revising these fits, we realized that some of the RVs they used were affected by embedded emission (likely resulting from the night sky) and are hence not reliable. By excluding these RV values, UCAC2 46706450-A does not show $\mathrm{RV}$ variation, in agreement with the results obtained here.

The WD spectrum is blueshifted by $-10 \pm 8 \mathrm{~km} \mathrm{~s}^{-1}$. Considering the gravitational redshift of $v_{\text {grav }}=8.6 \pm 1.3 \mathrm{~km} \mathrm{~s}^{-1}$ gives a RV value for the WD of $v_{\text {rad }}=-18.6 \pm 8.1 \mathrm{~km} \mathrm{~s}^{-1}$ (calculated using the mass and radius from Sect. 6). This agrees very well with the most precisely measured RV value of the $\mathrm{K}$ star $\left(v_{\text {rad }}=-18.35 \pm 0.07 \mathrm{~km} \mathrm{~s}^{-1}\right.$ from ASPCAP) and can be seen as a first hint that the system is indeed a physically connected binary and that the velocities of both stars represent the system velocity.

We stress, however, that with the current set of RV measurements we are only able to detect RV amplitudes larger than $\approx 15 \mathrm{~km} \mathrm{~s}^{-1}$, and that in case of a low inclination angle or a long orbital period, the amplitude can be much smaller than this.

\section{Distance and Galactic population membership}

The distance of UCAC2 46706450-A (the dominant source in the optical) based on the Gaia DR2 parallax is provided in the Bailer-Jones et al. (2018) catalog and amounts to $d=1417_{-43}^{+45} \mathrm{pc}$. From this we calculate a height above the Galactic plane of $z=1154_{-35}^{+36} \mathrm{pc}$, that is to say we find that it lies in a region where the thick disk dominates.

The Galactic population membership of UCAC2 46706450 can be further constrained by looking at the chemistry of UCAC2 46706450-A, as well as the kinematics of the system. Both thick disk and thin disk stars are observed over a wide metallicity range $([\mathrm{Fe} / \mathrm{H}]$ between -0.8 and 0.2 dex, Kordopatis et al. 2015), but thick disk stars are found to be more $\alpha$-rich at a given metallicity compared to thin disk stars (e.g., Fuhrmann 1998; Recio-Blanco et al. 2014). At metallicities above $[\mathrm{Fe} / \mathrm{H}]=0.1 \mathrm{dex}$, the separation of the two populations is, however, unclear (e.g., Hayden et al. 2017). At the average values $[\mathrm{Fe} / \mathrm{H}]=0.09$ dex (standard deviation $0.10 \mathrm{dex}$ ) and $[\alpha / \mathrm{M}]=0.02 \mathrm{dex}$ (standard deviation $0.07 \mathrm{dex}$ ) reported for UCAC2 46706450-A in the LAMOST and ASPCAP catalogs, the star appears just on the borderline between the two populations (see, e.g., Fig. 1 in Hayden et al. 2017). Therefore, it is worth also looking at the kinematics of the system. Since LAMOST, APOGEE, TNT, Palomar, Gaia, and HST/COS spectra do not indicate significant RV variations, we adopt the mean RV $(-21.95 \pm 4.55$, where the error represents the standard deviation of the individual measurements) as system velocity. With this and the proper motion and parallax from the Gaia DR2, we calculate space velocities of $U=46.4 \pm 1.0 \mathrm{~km} \mathrm{~s}^{-1}$, $V=-21.8 \pm 2.5 \mathrm{~km} \mathrm{~s}^{-1}$, and $W=-23.6 \pm 3.7 \mathrm{~km} \mathrm{~s}^{-14}$. The kinematic Galactic population membership can then be derived on the basis of the Mahalanobis distance, which is the

\footnotetext{
3 Geier et al. (2015) considered objects with $\log p>4.0$ as significantly variable, and objects with $-1.3>\log p>-4.0$ as possibly variable.

4 Cardinal directions, with $U$ being positive toward the Galactic center. Values have been adjusted for the Solar motion using the numbers of Tian et al. (2015).
}

number of standard deviations between the space velocities of UCAC2 46706450 and the velocity ellipsoids of the thin disk, thick disk, and the halo (Gianninas et al. 2015). Using the velocity ellipsoid values from Kordopatis et al. (2011), we find that the kinematics of UCAC 246706450 clearly point to membership of the thick disk. A final, and probably most decisive, criterion that can be used to determine the Galactic population membership is the age of the system, and this is discussed further in the next section.

\section{Radii, luminosities, masses, and ages}

In order to determine the radii of the two stellar components, we performed a fit to the spectral energy distribution (SED), by varying their solid angles $\pi(R / d)^{2}$ (which relates the astrophysical flux on the surface of the stars to what is received on Earth) until a good agreement of the combined synthetic flux and the observations was found. We assumed a reddening of $E_{\mathrm{B}-\mathrm{V}}=0.03$ (see Sect. 2) and the distance provided by Bailer-Jones et al. (2018) based on the Gaia parallax. For UCAC2 46706450-B, we used our best fit model from Sect. 2. For UCAC2 46706450-A, we used a NextGen model (Allard et al. 2012) with $T_{\text {eff }}=4950 \mathrm{~K}$, $\log g=3.0$, and a metallicity of $[\mathrm{Fe} / \mathrm{H}]=0$ dex, which is close to the average values of the derived parameters from various analyses. The errors on the radii were determined taking into account the uncertainties of the effective temperatures and the distance given by Bailer-Jones et al. (2018).

Our best fit is shown in Fig. 7. The black dots indicate filteraveraged fluxes that were converted from observed magnitudes. GALEX $F U V$ and $N U V$ magnitudes were taken from Bianchi et al. (2014) and converted to fluxes as outlined in Reindl et al. (2016). $B, V, g, r$, and $i$ magnitudes were taken from Henden et al. (2015), 2MASS $J, H$, and $K$ magnitudes from Cutri et al. (2003). To convert these magnitudes into fluxes, we used the zero-points provided in Holberg \& Bergeron (2006). Wide-field Infrared Survey Explorer (WISE) $W 1, W 2, W 3$, and $W 4$ magnitudes were taken from Cutri et al. (2003) and converted to fluxes using the zero-points and the flux corrections for a K2V star provided in Wright et al. (2010). The light blue, green, and red lines in Fig. 7 indicate the SEDs of UCAC2 46706450-B, UCAC2 46706450-A, and the combined best fit model, respectively. All model fluxes are corrected for interstellar reddening, using the reddening law of Fitzpatrick (1999) with $E_{\mathrm{B}-\mathrm{V}}=0.03$. The gray lines in Fig. 7 correspond to the HST/COS, co-added LAMOST, and APOGEE spectra. Overall, the combined model flux reproduces the observed SED well. Only the observed NUV flux exceeds the predicted flux by about 0.1 dex. We attribute this to the chromospheric flux of the K-type star, as chromospheric fluxes are known to exceed the photospheric flux by several orders of magnitude in chromospherically active stars (Stelzer et al. 2013, 2016; Dixon et al. 2020). Based on the NUV excess of $0.1 \mathrm{dex}$, we estimate that chromospheric flux of the K-type star exceeds its photospheric flux by about two orders of magnitude and which is also what is found for other such rapidly rotating and chromospherically active giants (Dixon et al. 2020). The WISE $W 3$ and $W 4$ fluxes also indicate a slight excess, but these bands are in particular sensitive to the flux corrections (gray open circles in Fig. 7 indicate WISE fluxes for which the flux correction was not taken into account). Thus, we regard the far-IR excess as uncertain.

For UCAC2 46706450-A, we derive a radius of $R_{A}=5.9_{-0.5}^{+0.7} R_{\odot}$, and for the hot WD, $R_{B}=0.040_{-0.004}^{+0.005} R_{\odot}$. Using $L=4 \pi \sigma R^{2} T_{\text {eff }}^{4}$, where $\sigma$ is the Stefan-Boltzmann constant, we 


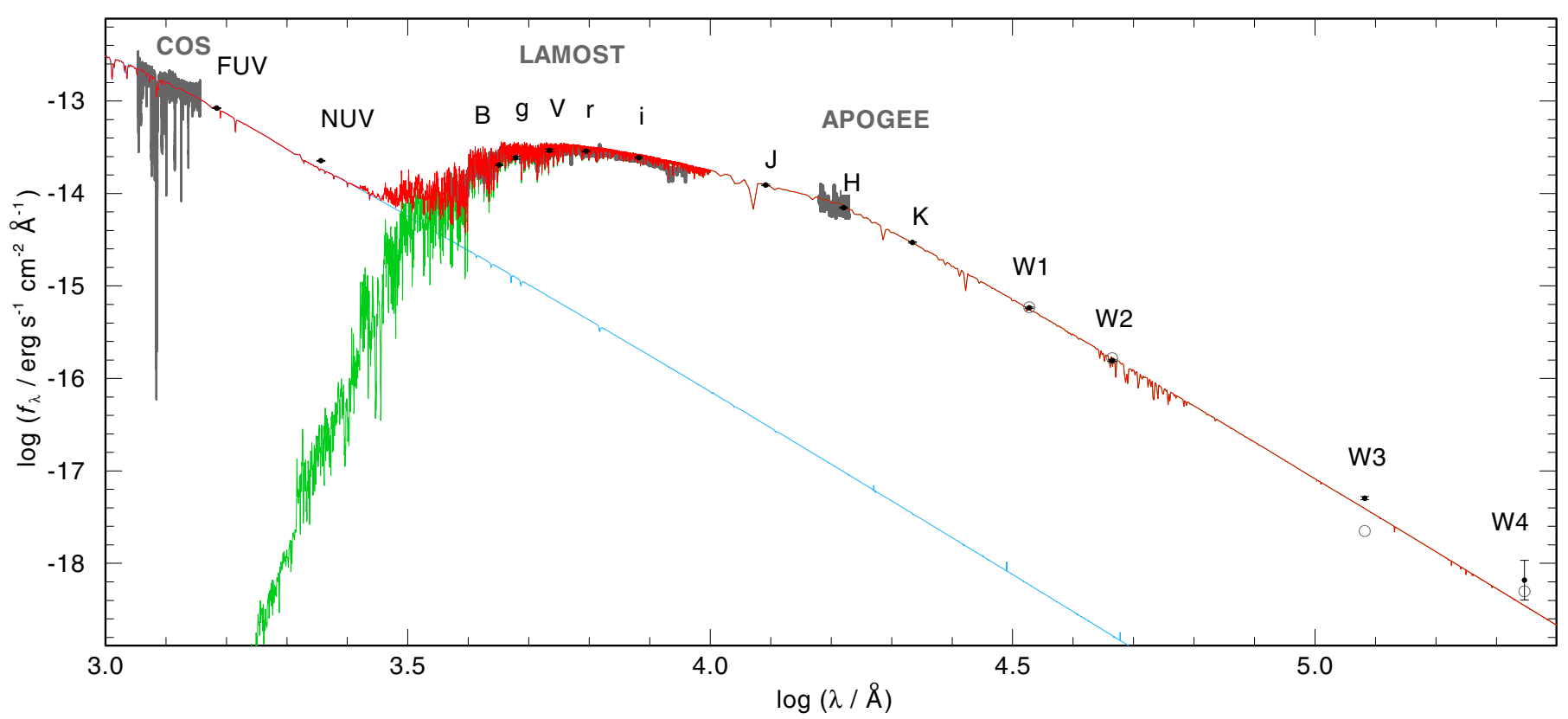

Fig. 7. Fit to the SED of UCAC2 46706450. HST/COS, LAMOST (normalized to $r$-band magnitude), and APOGEE spectra are shown in gray. The model fluxes from the hot WD and the K-type subgiant are shown in light blue and green, respectively. The combined model flux is shown in red. The black dots indicate filter-averaged fluxes that were converted from observed magnitudes. Gray open circles indicate WISE fluxes for which the flux correction was not taken into account.

calculate luminosities of $L_{A}=19_{-5}^{+5} L_{\odot}$ and $L_{B}=176_{-49}^{+55} L_{\odot}$ for UCAC2 46706450-A and UCAC2 46706450-B, respectively. These values are what is expected for a subgiant star and a hot WD that is just about to enter the WD cooling sequence. Hence, we conclude that the system is indeed a physical binary.

With the spectroscopically determined surface gravities (average value for the $\mathrm{K}$ star from Table 2) and using $M=g R^{2} / G$, where $G$ is the gravitational constant, we calculate masses of $M_{A}=1.4_{-0.7}^{+1.2} M_{\odot}$ and $M_{B}=1.5_{-1.1}^{+4.4} M_{\odot}$ for the K-type subgiant and the hot WD, respectively. We note that the large errors on the masses are mainly a consequence of large uncertainties of the surface gravities of both stars. The masses can be constrained more precisely when we compare their locations in the Hertzsprung Russell diagram (HRD) with predictions from stellar evolutionary calculations. But this mass determination also has its limitations, as is outlined in the following. In the upper panel of Fig. 8, we show the location of UCAC2 46706450-A in the HRD compared to stellar evolutionary tracks of Pietrinferni et al. (2004). The tracks are calculated for $Z=0.01([\mathrm{Fe} / \mathrm{H}]=0-0.25 \mathrm{dex})$ and a helium mass fraction of 0.259 (upper panel in Fig. 8) and suggest a mass of $M_{A}=1.2_{-0.4}^{+0.7} M_{\odot}$. In the lower panel of Fig. 8, we show the evolutionary tracks of Pietrinferni et al. (2004), which were calculated for a slightly higher metallicity of $Z=0.03([\mathrm{Fe} / \mathrm{H}]=0.26 \mathrm{dex})$ and a helium mass fraction of 0.288 . These tracks are shifted toward higher effective temperatures, and thus suggest a higher mass of $M_{A}=1.9_{-0.9}^{+0.5} M_{\odot}$. This shows that the mass of the Ktype subgiant obtained from the HRD depends significantly on the chemistry used in the evolutionary calculations. We therefore infer a possible mass range of $M_{A}=0.8-2.4 M_{\odot}$ based on these evolutionary models.

In Fig. 9, we show the location of UCAC2 46706450-B in the HRD compared to evolutionary calculations of H-shell burning post-asymptotic giant branch (AGB) stars from Miller Bertolami (2016). This suggests a mass of $M_{B}=0.54 \pm 0.02 M_{\odot}$ and that the surface gravity of the WD might be rather at the lower boundary of the error margin $(\log g=7.0 \pm 0.1)$ determined spectroscopically. In gray, a He-shell burning very late thermal pulse (VLTP) evolutionary track is shown. This track extends toward higher $T_{\text {eff }}$ at the luminosity found for UCAC2 46706450-B, which supports the assumption that the WD is indeed H-rich (higher envelope mass; much lower mass not possible due to the limited age of the disk/Milky Way). In Fig. 9, we also indicate the initial masses assumed for the computation of the evolutionary tracks. This suggests that if the initial-to-final mass relation (IFMR) assumed in these calculations are accurate and the system has not interacted in previous evolutionary phases, the initial mass of the hot WD was about $1 M_{\odot}$, or only slightly higher. A low mass of the WD is also supported by the absence of a PN. The tracks of Miller Bertolami (2016) calculated for $Z=0.01$ predict a postAGB age of about $72 \mathrm{kyrs}$ for the $0.52 M_{\odot}$ model and only about $10 \mathrm{kyrs}$ for the $0.56 M_{\odot}(Z=0.01)$ model. This means that if UCAC2 46706450-B should have ejected a PN on the AGB, and we assume that a PN is typically visible for $30 \mathrm{kyrs}$, then, in the case of a low mass post-AGB star, the PN should have faded away already, while for higher masses, the PN should still be visible. Radii, luminosities, and masses of UCAC2 46706450-A and UCAC2 46706450-B are summarized in Table 2.

If we assume again that the system has not undergone mass transfer in its past and the initial mass of the WD was between 1.0 and $1.25 M_{\odot}$, this would imply that the initial mass of UCAC2 $46706450-A$ is only slightly lower than this. The total lifetime of stars in that mass range at solar metallicity stated by Miller Bertolami (2016) lies between 5.3 and 12.5 Gyrs. Thus, it is entirely possible that the system indeed belongs to the thick disk, which is generally found to be exclusively old, meaning older than nine Gyrs (Feltzing \& Bensby 2009; Kilic et al. 2017; Hayden et al. 2017). We also note that in the course of their study of main-sequence turnoff and subgiant stars from the AMBRE:HARPS survey, Hayden et al. (2017) find stars at the metallicity of UCAC2 46706450-A that have ages between 
K. Werner et al.: An extremely hot white dwarf with a rapidly rotating K-type subgiant companion

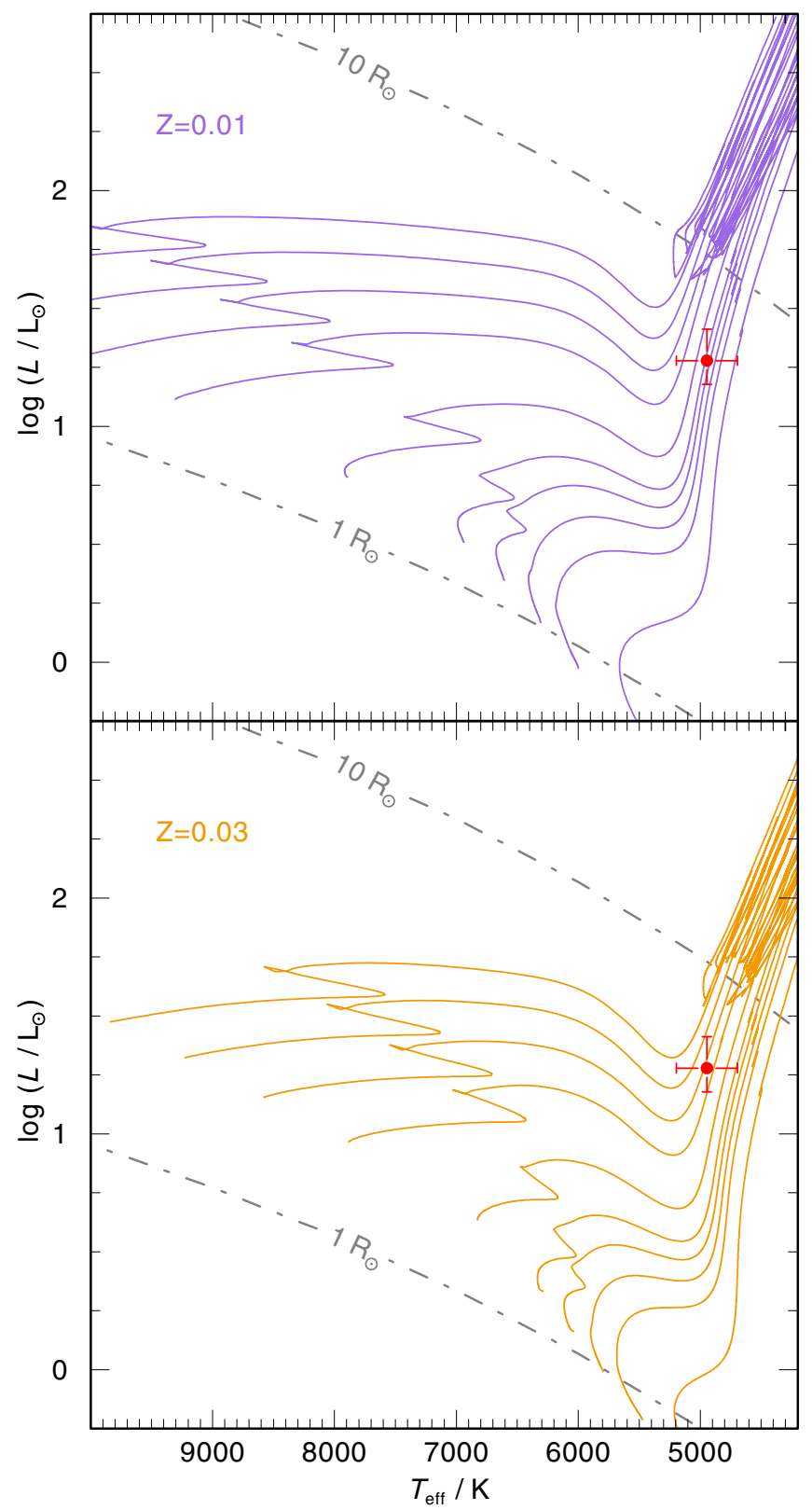

Fig. 8. Location of UCAC2 46706450-A (red) in the HRD compared to stellar evolutionary tracks of Pietrinferni et al. (2004) for initial masses of $0.8,1.0,1.1,1.2,1.3,1.5,1.8,2.0,2.2$, and $2.4 M_{\odot}$. Upper panel: evolutionary tracks calculated for $Z=0.01([\mathrm{Fe} / \mathrm{H}]=0.25 \mathrm{dex})$ and a He mass fraction of 0.259. Lower panel: tracks calculated for $Z=0.03([\mathrm{Fe} / \mathrm{H}]=0.26 \mathrm{dex})$ and a He mass fraction of 0.288 . The gray dashed-dotted lines indicate radii of 1 and $10 R_{\odot}$.

8 and 10 Gyrs. However, since the IFMR is poorly constrained at the low-mass end, and mass loss on the RGB is not well understood, it is not possible to give a more precise estimate of the system's age.

\section{Light curve variability}

We obtained the Catalina Sky Survey (CSS, Drake et al. 2009) DR2 $V$-band light curve of UCAC2 46706450 as well as $c$ - and $o$-band light curves (effective wavelengths 0.53 and $0.68 \mu \mathrm{m}$, respectively) from the Asteroid Terrestrial-impact Last Alert System (ATLAS, Tonry et al. 2018) DR1 (Fig. 10).

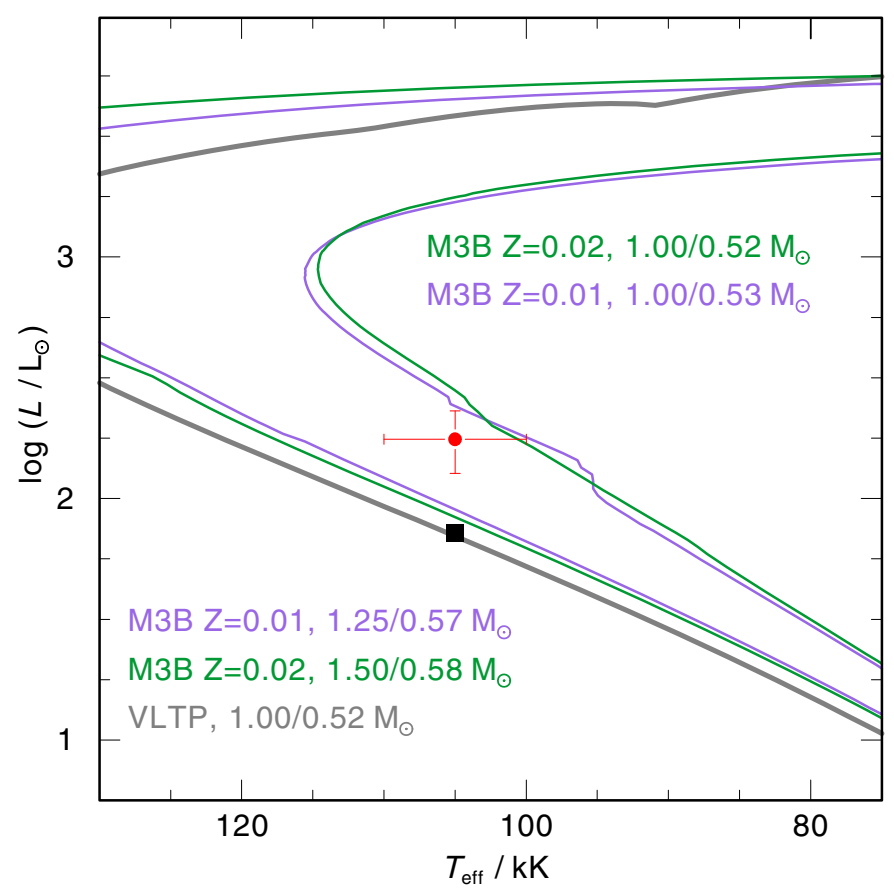

Fig. 9. Location of UCAC2 46706450-B (red) in the HRD compared to stellar evolutionary tracks of Miller Bertolami (2016) calculated for $Z=0.01$ (purple) and $Z=0.02$ (green). Initial and final masses of the tracks are indicated. Shown in gray is a VLTP evolutionary track. The black square indicates the position of EGB 6.

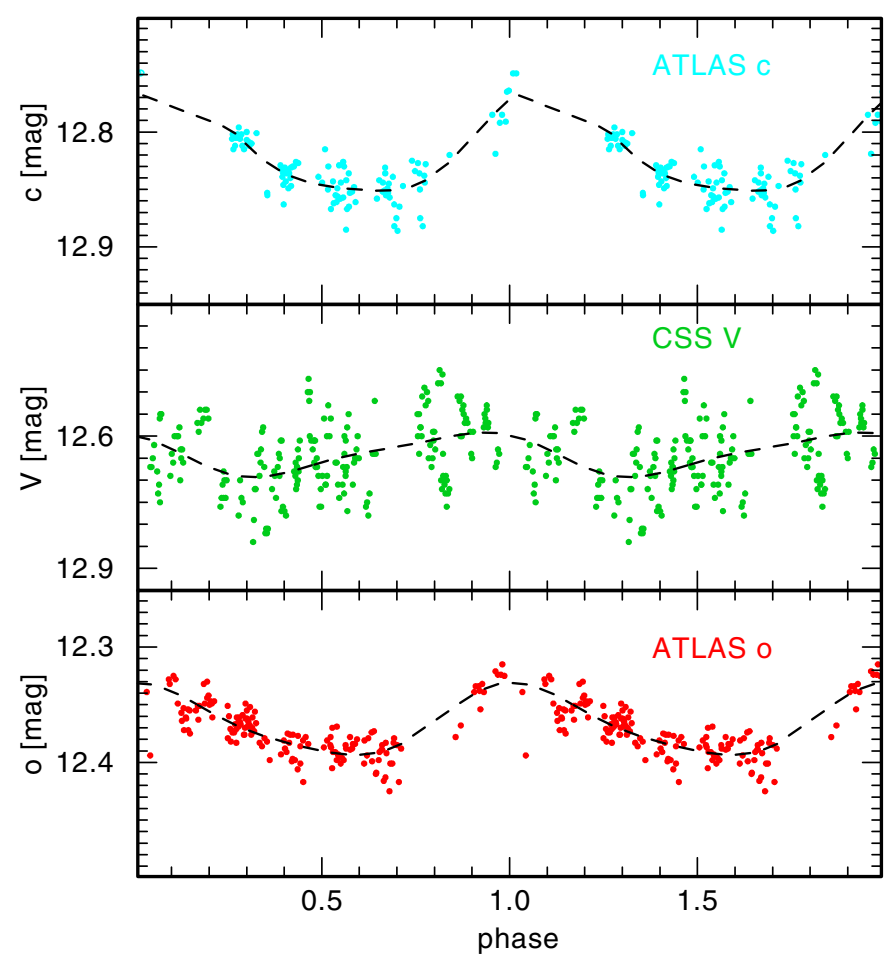

Fig. 10. ATLAS $c$-band, CSS $V$-band, and ATLAS $o$-band phase-folded light curves. The CSS light curve is folded with the $1.97861678 \mathrm{~d}$ period.

We also observed the light curve of UCAC2 46706450 in the $V$-band using the Tübingen $80 \mathrm{~cm} \mathrm{f/8} \mathrm{telescope} \mathrm{on} \mathrm{April} \mathrm{14,} \mathrm{15,}$ and 16 (Fig. 11). The images were taken with an integration time of $120 \mathrm{~s}$ using a SBIG STL-1001 CCD camera and a binning of $2 \times 2$ pixels. Almost every night, data could be obtained 

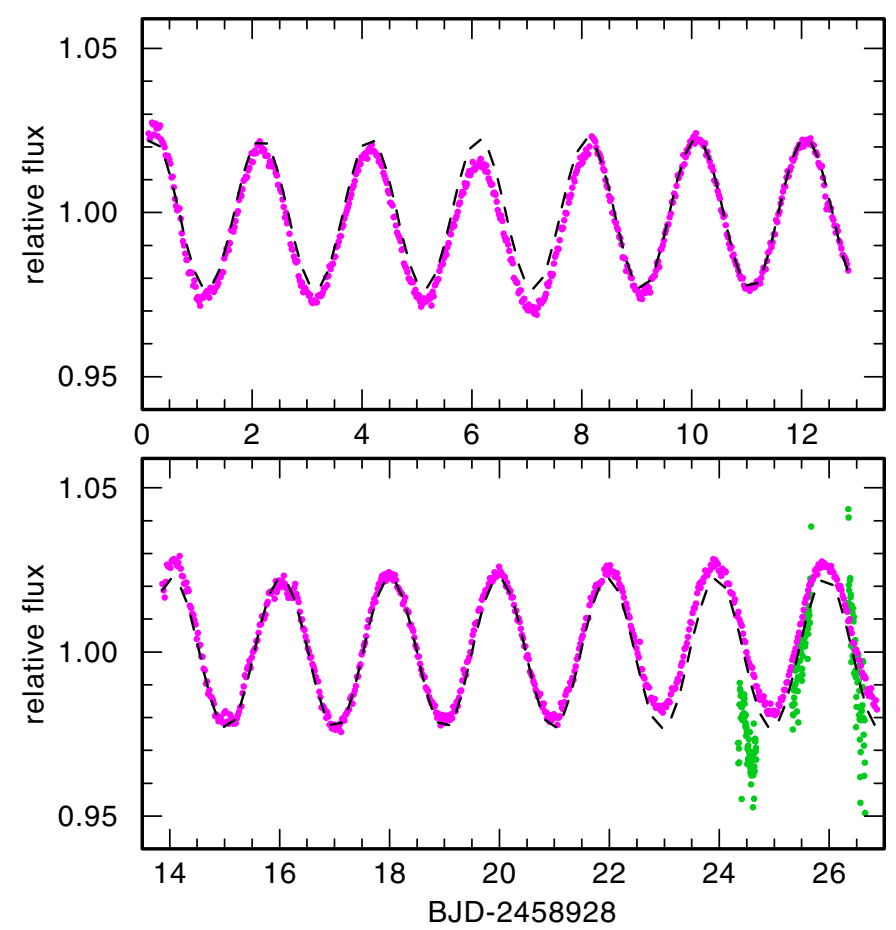

Fig. 11. TESS (magenta) and Tübingen $80 \mathrm{~cm}$ telescope $V$-band (green) light curves. The $V$-band light curve has been shifted by one period.

over seven hours. The sky was clear during the first two nights, whereas thin cirrus was passing throughout the third night. All images were background and flatfield corrected using the IDL software Time Resolved Imaging Photometry Package (TRIPP, Schuh et al. 2003), which was also used to perform aperture photometry. The flux error was minimized by testing different aperture radii with respect to comparison star variances. We note that the data were obtained under non-photometric conditions, with the weather conditions being worst in the third night. As there is only one equally bright star in the field of view, we used only one comparison star for the first and third nights. During the second night, the observing conditions were best, so a second comparison star could be used.

In addition, we used Transiting Exoplanet Survey Satellite (TESS) observations of UCAC2 46706450 (Fig. 11). The star was in the TESS field of view during Sector 23 observations. As there is no 2-min cadence light curve available, we performed photometry using the 30-min cadence full-frame images (FFIs) using LIGHTKURVE (Lightkurve Collaboration 2018). We used a relatively small $4 \times 4$ pixel aperture to minimize contamination from two nearby stars, and subtracted a background estimated using the same aperture on a nearby region with no sources.

For the analyses of the light curves, we used the VARTOOLS program (Hartman \& Bakos 2016) to perform a generalized Lomb-Scargle search (Zechmeister \& Kürster 2009; Press et al. 1992) for periodic sinusoidal signals. For the ATLAS $c$ and $o$-bands, we find the strongest signal at $P=1.97913923 \mathrm{~d}$ and $P=1.97899913 \mathrm{~d}$, with associated false alarm probabilities (FAPs) of $10^{-37}$ and $10^{-28}$, respectively. The derived period from the $o$-band equals exactly half the period given in Heinze et al. (2018), who classified the light curve of UCAC2 46706450 as an irregular variable. This class serves in their catalog as a "catch-all" bin for objects that do not seem to fit into any of their more specific categories. We also performed a generalized Lomb-Scargle search on the CSS light curve, and find the strongest signal at $P=0.21064926 \mathrm{~d}$, which, however, has a much higher FAP of $10^{-13}$. A possible explanation could be that UCAC2 46706450 is indeed an irregular variable and that its photometric period and amplitude change over time. Thus, in the much longer CSS light curve (time coverage $\approx 8 \mathrm{yr}$ ) no clean signal can be detected. Another point to notice for the CSS light curve is that during MJD = 53550 and $\mathrm{MJD}=53901$, UCAC2 46706450 underwent a brightness variation of 0.7 mag. Using only CSS data obtained after MJD $=54000$, we repeated the generalized Lomb-Scargle search by looking only for periods between 1.9 and $2.1 \mathrm{~d}$. By that, we find a significant peak at $P=1.97861678$ (FAP $=10^{-6}$ ), close to the value found for the ATLAS light curves. In Fig. 10, we show the phase-folded ATLAS $c$ - and $o$-band and CSS light curves. The black dashed lines are fits of a harmonic series to the phase-folded light curves (see Eq. (49) in Hartman \& Bakos 2016) and predict peak-topeak amplitudes of $0.08,0.10$, and $0.06 \mathrm{mag}$ in the $V$-, $c$ - and $o$-bands, respectively. We note, however, that due to the photometric uncertainties (0.05 mag for CSS, and $0.01 \mathrm{mag}$ for the ATLAS light curves), the differences in the amplitudes might not be real.

For the TESS light curve, we derive a period of $1.98063847 \mathrm{~d}$ $\left(\right.$ FAP $\left.=10^{-755}\right)$. The light curve is shown in Fig. 11 along with the V-band light curve obtained with the Tübingen $80 \mathrm{~cm}$ telescope, which is shifted for one period. We find that the phases of both light curves match nicely, though the amplitudes differ. We note, however, that the amplitudes of both should be treated with caution. As mentioned above, the Tübingen $80 \mathrm{~cm}$ telescope light curve was obtained under non-photometric conditions, and due to the large pixel-size of TESS two neighboring nearby stars may add additional continuum flux, which in turn may reduce the amplitude of the TESS light curve. What can also be seen in Fig. 11 is that the shape of the TESS light curve changes slightly, which is typical for star spot evolution (Kővári et al. 2019).

If we assume the observed period of the light curve variation corresponds to an orbital period, and a mass of $0.54 M_{\odot}$ for the WD and $1.0 M_{\odot}$ of the K-type subgiant (Sect. 6), then we calculate via the third Kepler law, a separation of the two stars of $a=7.7 R_{\odot}$. In that case, a strong reflection effect should be noticeable. In such systems, a tidally-locked cool companion is strongly irradiated by the hot primary, also causing sinusoidal light curve variations at the orbital period of the system. Then, however, the amplitudes of the light curve variations should be increasing toward longer wavelengths, which is not observed. In addition, we would expect to see all Balmer lines, the CNO line complex around $4560 \AA$, and possibly also some other lines in emission. These two observational properties are seen in BE UMa, which also has a $T_{\text {eff }}=105000 \mathrm{~K}$ hot primary, an orbital period of $2.29 \mathrm{~d}$, and a distance $a=8.6 R_{\odot}$ from its cool companion (Ferguson \& James 1994) and in many other reflection effect systems containing a very hot primary (e.g., Sing et al. 2004; Exter et al. 2005; Nagel et al. 2006; Shimansky et al. 2015; Mitrofanova et al. 2016).

Thus, we conclude that the variations are caused by spots on the surface of the star and that the photometric period corresponds to the rotational period of UCAC2 46706450-A. With a radius of $5.9_{-0.5}^{+0.7} R_{\odot}$ and rotation period of $1.98 \mathrm{~d}$, we calculate a rotational velocity of $v_{\text {rot }}=151_{-13}^{+18} \mathrm{~km} \mathrm{~s}^{-1}$. If we assume that the mass is close to $1 M_{\odot}$ (Sect. 6$)$, this value is close to the breakup velocity ${ }^{5}$. With $v \sin (i)=80.76 \mathrm{~km} \mathrm{~s}^{-1}$ as suggested

\footnotetext{
5 The breakup velocity $v_{\text {breakup }}=\sqrt{G M / R}$ of a $1.0 / 2.0 M_{\odot}$ star with a radius of $5.9 R_{\odot}$ is $180 / 254 \mathrm{~km} \mathrm{~s}^{-1}$, respectively.
} 
by the ASPCAP fit of the APOGEE spectrum, one can estimate that the inclination of rotational axis is $i=32^{\circ}$.

If we assume a bound rotation (as seen in many RS CVn stars), that the inclination angle of the rotational axis is perpendicular to the orbital plane, and the masses given in Table 2, then RV semi-amplitudes of $58-105 \mathrm{~km} \mathrm{~s}^{-1}$ and $23-41 \mathrm{~km} \mathrm{~s}^{-1}$ would be expected for the WD and subgiant, respectively. The latter is much larger than the standard deviation of the individual RV measurements of UCAC2 46706450-A $\left(4.55 \mathrm{~km} \mathrm{~s}^{-1}\right.$, see Sect. 5) and, thus, again speaks against a close binary system.

\section{Conclusions}

From the SED fit, the fact that we do not find significant RV variability, that the RVs of UCAC2 46706450-A and UCAC2 46706450-B agree, and that no reflection effect is noticeable in the light curves and spectra, we conclude the system is most likely a wide, physically connected binary system. UCAC2 46706450-A is a subgiant just about to climb up the RGB, while UCAC2 46706450-B is an extremely hot WD just about to enter the WD cooling sequence.

Kinematically, and from its height above the Galactic plane, the system belongs to the Galactic thick disk. This can also explain the low mass of the WD without the need for binary interactions, as the thick disk is generally found to contain exclusively old, and by that low-mass stars.

\subsection{The white dwarf}

We determined the atmospheric parameters of the hot WD in the binary UCAC2 46706450. Within error limits, the light metal (C, $\mathrm{N}, \mathrm{O}, \mathrm{F}, \mathrm{Si}, \mathrm{S}$ ) abundances are solar, whereas iron-group elements (Cr, Mn, Fe, Ni) are slightly enhanced (0.45-0.70 dex oversolar). The $\mathrm{H} / \mathrm{He}$ ratio cannot be determined from the available spectra. However, by comparison with another WD we can conclude that it is likely a DAO white dwarf. The effective temperature $\left(T_{\text {eff }}=105000 \mathrm{~K}\right)$ is the same as that of EGB 6, a DAO central star of a PN (Werner et al. 2018b). The metal abundance pattern of EGB 6 is very similar, and its $\mathrm{H} / \mathrm{He}$ ratio is solar (Fig. 4, right panel). It was concluded that EGB 6 is at the wind limit of its cooling track, shortly before gravitational settling and radiative acceleration of elements begin to affect the photospheric abundance pattern. Before the wind limit, a radiation-driven wind prevents atomic diffusion (e.g., Unglaub \& Bues 2000). Thus, UCAC2 46706450-B is probably a DAO white dwarf. The He abundance could be subsolar since the slightly oversolar heavy-metal abundances indicate that diffusion began to act. On the other hand, heavy-metal abundances in the K-type subgiant are also oversolar (Fig. 4, left panel), thus, the element abundance pattern probably reflects the initial metallicity of both stars. UCAC2 46706450-B could be just crossing the wind-limit. Observational evidence testifies that the wind limit for H-rich WDs is indeed located at $T_{\text {eff }} \approx 105000 \mathrm{~K}$ (Werner et al. 2018b, 2019) and recent theoretical work confirms this result (Krtička et al. 2020).

We emphasize, however, that we cannot rule out that the WD is a helium-rich DO white dwarf. Before the wind limit, DOs can also exhibit a near-solar metal abundance pattern (Werner et al. 2017). To make a conclusive decision, an observation covering the position of He II $1640 \AA$ is needed. We can exclude that the WD belongs to the non-DA PG1159 class whose members have very abundant $\mathrm{C}$ and $\mathrm{O}$ (Werner \& Herwig 2006).

EGB 6 is slightly more massive $\left(M=0.58_{-0.04}^{+0.12} M_{\odot}\right.$, Fig. 9) than UCAC2 46706450-B $\left(M=0.54 \pm 0.02 M_{\odot}\right)$, and thus
EGB 6 evolved from the AGB to its current position more rapidly (post-AGB time is about $\log \left(t_{\text {evol }} \mathrm{yr}^{-1}\right)=3.60_{-0.09}^{+1.26}$ ) than UCAC2 46706450-B $\left(\log \left(t_{\text {evol }} \mathrm{yr}^{-1}\right) \approx 4.86\right)$. This might explain why for EGB 6 the PN is still visible, while for UCAC2 46706450-A no PN can be detected.

\subsection{The $K$ subgiant}

We reported the discovery of a time-variable emission of $\mathrm{H} \alpha$ as well as emission line cores in the $\mathrm{Ca}$ II $\mathrm{H}$ and $\mathrm{K}$ doublet, which is a strong hint to chromospheric activity. Further evidence for chromospheric activity can be seen from the NUV excess. Furthermore, we found that the object is photometrically variable with a period of $\approx 2 \mathrm{~d}$ only. The TESS light curve shows small variations in the shape of light curve. Hence, we conclude that the variations are caused by (evolving) spots on the surface of UCAC2 46706450-A. With the radius from the SED fit, we find that the star is rotating extremely fast with $v_{\text {rot }}=151_{-13}^{+18} \mathrm{~km} \mathrm{~s}^{-1}$. This marks UCAC2 46706450-A as one of the most rapidly rotating subgiants known.

The chromospheric activity, NUV excess flux, star spots, and very rapid rotation is what is also seen in FK Comae Berenices (FK Com) stars, a small group (only a handful of objects known) of single, rapidly rotating, and very active GK-type subgiants, which are believed to be the product of a recent merger (Eggen \& Iben 1989). The prototype FK Com is the undisputed "king of spin" amongst these single red giants with an equatorial spin velocity of $v_{\mathrm{eq}}=179 \mathrm{~km} \mathrm{~s}^{-1}$, followed by HD 199178, the so called "spin vice king" amongst giant stars (Costa et al. 2015), with $v_{\mathrm{eq}}=93 \mathrm{~km} \mathrm{~s}^{-1}$ (Strassmeier 2009). A related class is that of the more frequently known RS Canum Venaticorum (RS CVn) variable stars, which are close binary systems consisting of a chromospherically active subgiant component, which exhibits brightness variations caused by large cool spots (Hall 1972), and which is also known to be fast rotating (Strassmeier 2009). There are also two other hot (pre-) WDs known, which are in a binary system with chromospherically active and rapidly rotating cool companions: LW Hya and LoTr 5. The latter is a wide binary in a highly eccentric orbit $(e \approx 0.3, P=2700 \mathrm{~d}$, Jones et al. 2017) consisting of a hot pre-WD or WD, and the cool component, IN Com, is a rapidly rotating $\left(v_{\mathrm{eq}}=95 \mathrm{~km} \mathrm{~s}^{-1}\right.$, Strassmeier 2009) and magnetically active G5 III giant that also shows $\mathrm{H} \alpha$ lineprofile variations (Kővári et al. 2019). LW Hya is a resolved binary system composed of a hot DAO WD (the ionizing star of A 35, Ziegler et al. 2012; Löbling et al. 2019) and a magnetically active G8 III-IV-type companion $\left(v_{\mathrm{eq}}=127 \mathrm{~km} \mathrm{~s}^{-1}\right.$, Strassmeier 2009). For LW Hya, it is, however, not clear if the system is really a physical binary, due to the mismatch of the spectroscopic distance of the WD and the optical parallax.

The driving mechanism for the chromospheric activity and the reason for the rapid rotation observed in UCAC2 46706450-A remains to be explored. In main sequence stars of spectral type F, G, K, and early M, a self-sustaining magnetic dynamo driven by rotation and convection is believed to be the source of chromospheric activity. Since due to magnetic braking, the rotational velocity of the star is expected to decrease with age, the chromospheric activity is thought to decrease as well. However, if angular momentum is sustained, by tidal interaction as in the case of short-period binaries (this holds for the RS CVn stars), or maintained by convection, chromospheric activity can be preserved (Zhao et al. 2011).

Besides a recent stellar merger, sudden dredge-up of angular momentum from the stellar interior (Simon \& Drake 1989), and accretion of a substellar companion (Peterson et al. 1983; 
Carlberg et al. 2012) are considered as possible explanations for the abnormally high rotation rates of FK Com stars. As mentioned before, the lack of significant RV variations makes the close-binary scenario for UCAC2 46706450 very unlikely. One may therefore speculate that the system used to be a hierarchical triple system, and that UCAC2 46706450-A was produced by the merger of the inner binary, as was suggested for IN Com (Jasniewicz et al. 1987; Malasan et al. 1991).

\subsection{Has UCAC2 46706450 an ultra-wide companion?}

Recently, Tian et al. (2020) reported that based on common Gaia parallaxes and proper motions, UCAC2 46706450 is in an ultra-wide binary system with Gaia DR2 1391040916768689280 (alias SDSS J152844.16+430417.1), separated by 75.48951" (111 $634 \mathrm{AU})$. This fainter star has an absolute Gaia $G$-band magnitude of $M_{G}=6.76 \mathrm{mag}$ and a color index of $G_{\mathrm{BP}}-$ $G_{\mathrm{RP}}=1.26$ (Tian et al. 2020), thus it is likely a K-type mainsequence star. That implies a low mass $\left(M \leq 0.8 M_{\odot}\right)$ of the possible ultra-wide companion, which would also be in line with the lower limit of the (initial) masses of UCAC2 46706450-A and UCAC2 46706450-B, meaning that they indeed could have formed at the same time.

\subsection{Prospects}

A future detailed spectral analysis of UCAC2 46706450-A, which considers all spectral observations at once and also considers the flux contribution of the hot WD in the blue, would be highly desirable. This would help to better constrain the chemical composition and the mass of the star, as well as the age of the system. Near-UV spectroscopy could help to determine the helium abundance of the WD and to investigate the nature of the NUV excess flux. Long-term, high-resolution monitoring, that allows RVs to be measured with an accuracy of a few hundred $\mathrm{m} \mathrm{s}^{-1}$, will help to constrain the orbital period of the system and in turn makes it possible to conclude if the system underwent any mass transfer or wind accretion in its past, and to check if the merger scenario could be possible. Spectroscopic followup of SDSS J152844.16+430417.1 would allow us to verify if it is an ultra-wide companion by testing if its $\mathrm{RV}$ and chemical composition agree with UCAC2 46706450-A.

Acknowledgements. We thank the referee, Uli Heber, for his constructive report We appreciate useful discussions with M3B and Stephan Geier. We thank Matti Dorsch and Boris Gänsicke for pointing out this system to us and useful discussions. A.R.M. acknowledges support from the MINECO unde the Ramón y Cajal programme (RYC-2016-20254) and the AYA2017-86274-P grant, and the AGAUR grant SGR-661/2017. The TMAD tool (http://astro. uni-tuebingen. de/ TMAD) used for this paper was constructed as part of the activities of the German Astrophysical Virtual Observatory. This work has made use of data obtained at the Thai National Observatory on Doi Inthanon, operated by NARIT. Some of the data presented in this paper were obtained from the Mikulski Archive for Space Telescopes (MAST). This research has made use of NASA's Astrophysics Data System and the SIMBAD database, operated at CDS, Strasbourg, France. This research made use of Lightkurve, a Python package for Kepler and TESS data analysis (Lightkurve Collaboration 2018). This work has made use of data from the European Space Agency (ESA) mission Gaia (https://www.cosmos.esa.int/gaia), processed by the Gaia Data Processing and Analysis Consortium (DPAC, https://www.cosmos.esa.int/ web/gaia/dpac/consortium). Funding for the DPAC has been provided by national institutions, in particular the institutions participating in the Gaia Multilateral Agreement. This work has made use of BaSTI web tools. This work includes data from the Asteroid Terrestrial-impact Last Alert System (ATLAS) project. ATLAS is primarily funded to search for near Earth asteroids through NASA grants NN12AR55G, 80NSSC18K0284, and 80NSSC18K1575; byproducts of the NEO search include images and catalogs from the survey area. The ATLAS science products have been made possible through the contributions of the University of Hawaii Institute for Astronomy, the Queen's University
Belfast, the Space Telescope Science Institute, and the South African Astronomical Observatory. Guoshoujing Telescope (the Large Sky Area Multi-Object Fiber Spectroscopic Telescope LAMOST) is a National Major Scientific Project built by the Chinese Academy of Sciences. Funding for the project has been provided by the National Development and Reform Commission. LAMOST is operated and managed by the National Astronomical Observatories, Chinese Academy of Sciences. Funding for the Sloan Digital Sky Survey IV has been provided by the Alfred P. Sloan Foundation, the U.S. Department of Energy Office of Science, and the Participating Institutions. SDSS-IV acknowledges support and resources from the Center for High-Performance Computing at the University of Utah. The SDSS web site is www.sdss.org. SDSS-IV is managed by the Astrophysical Research Consortium for the Participating Institutions of the SDSS Collaboration including the Brazilian Participation Group, the Carnegie Institution for Science, Carnegie Mellon University, the Chilean Participation Group, the French Participation Group, Harvard-Smithsonian Center for Astrophysics, Instituto de Astrofísica de Canarias, The Johns Hopkins University, Kavli Institute for the Physics and Mathematics of the Universe (IPMU) / University of Tokyo, the Korean Participation Group, Lawrence Berkeley National Laboratory, Leibniz Institut für Astrophysik Potsdam (AIP), Max-Planck-Institut für Astronomie (MPIA Heidelberg), Max-Planck-Institut für Astrophysik (MPA Garching), Max-Planck-Institut für Extraterrestrische Physik (MPE), National Astronomical Observatories of China, New Mexico State University, New York University, University of Notre Dame, Observatário Nacional / MCTI, The Ohio State University, Pennsylvania State University, Shanghai Astronomical Observatory, United Kingdom Participation Group, Universidad Nacional Autónoma de México, University of Arizona, University of Colorado Boulder, University of Oxford, University of Portsmouth, University of Utah, University of Virginia, University of Washington, University of Wisconsin, Vanderbilt University, and Yale University.

\section{References}

Allard, F., Homeier, D., \& Freytag, B. 2012, Phil. Trans. R. Soc. London Ser. A, 370,2765

Andrews, J. J., Agüeros, M. A., Gianninas, A., et al. 2015, ApJ, 815, 63

Asplund, M., Grevesse, N., Sauval, A. J., \& Scott, P. 2009, ARA\&A, 47, 481

Bailer-Jones, C. A. L., Rybizki, J., Fouesneau, M., Mantelet, G., \& Andrae, R. 2018, AJ, 156, 58

Baxter, R. B., Dobbie, P. D., Parker, Q. A., et al. 2014, MNRAS, 440, 3184

Bianchi, L., Conti, A., \& Shiao, B. 2014, VizieR Online Data Catalog: II/335

Carlberg, J. K., Cunha, K., Smith, V. V., \& Majewski, S. R. 2012, ApJ, 757, 109

Casey, A. R., Hogg, D. W., Ness, M., et al. 2016, ArXiv e-prints [arXiv: 1603.03040 ]

Catalán, S., Isern, J., García-Berro, E., et al. 2008, A\&A, 477, 213

Chen, X., Han, Z., Deca, J., \& Podsiadlowski, P. 2013, MNRAS, 434, 186

Costa, A. D., Canto Martins, B. L., Bravo, J. P., et al. 2015, ApJ, 807, L21

Cutri, R. M., Skrutskie, M. F., van Dyk, S., et al. 2003, VizieR Online Data Catalog: II/ $/ 246$

Davis, P. J., Kolb, U., \& Willems, B. 2010, MNRAS, 403, 179

Dixon, D., Tayar, J., \& Stassun, K. G. 2020, AJ, 160, 12

Drake, A. J., Djorgovski, S. G., Mahabal, A., et al. 2009, ApJ, 696, 870

Duchêne, G., \& Kraus, A. 2013, ARA\&A, 51, 269

Eggen, O. J., \& Iben, I., J. 1989, AJ, 97, 431

Exter, K. M., Pollacco, D. L., Maxted, P. F. L., Napiwotzki, R., \& Bell, S. A 2005, MNRAS, 359, 315

Feltzing, S., \& Bensby, T. 2009, IAU Symp., 258, 23

Ferguson, D. H., \& James, T. A. 1994, ApJS, 94, 723

Fitzpatrick, E. L. 1999, PASP, 111, 63

Fuhrmann, K. 1998, A\&A, 338, 161

García Pérez, A. E., Allende Prieto, C., Holtzman, J. A., et al. 2016, AJ, 151, 144

Geier, S., Marsh, T. R., Wang, B., et al. 2013, A\&A, 554, A54

Geier, S., Kupfer, T., Heber, U., et al. 2015, A\&A, 577, A26

Gianninas, A., Kilic, M., Brown, W. R., Canton, P., \& Kenyon, S. J. 2015, ApJ, 812,167

Gray, R. O., \& Corbally, C., J. 2009, Stellar Spectral Classification (Princeton: Princeton University Press)

Hall, D. S. 1972, PASP, 84, 323

Han, Z., Podsiadlowski, P., Maxted, P. F. L., Marsh, T. R., \& Ivanova, N. 2002, MNRAS, 336, 449

Hartman, J. D., \& Bakos, G. Á. 2016, Astron. Comput., 17, 1

Hayden, M. R., Recio-Blanco, A., de Laverny, P., Mikolaitis, S., \& Worley, C. C. 2017, A\&A, 608, L1

Heinze, A. N., Tonry, J. L., Denneau, L., et al. 2018, AJ, 156, 241

Henden, A. A., Levine, S., Terrell, D., \& Welch, D. L. 2015, AAS Meeting Abstracts, 225, 336.16

Ho, A. Y. Q., Ness, M. K., Hogg, D. W., et al. 2017, ApJ, 836, 5

Holberg, J. B., \& Bergeron, P. 2006, AJ, 132, 1221 
Holberg, J. B., Oswalt, T. D., Sion, E. M., Barstow, M. A., \& Burleigh, M. R. 2013, MNRAS, 435, 2077

Jasniewicz, G., Duquennoy, A., \& Acker, A. 1987, A\&A, 180, 145

Jones, D., Van Winckel, H., Aller, A., Exter, K., \& De Marco, O. 2017, A\&A, 600, L9

Kővári, Z., Strassmeier, K. G., Oláh, K., et al. 2019, A\&A, 624, A83

Kilic, M., Munn, J. A., Harris, H. C., et al. 2017, ApJ, 837, 162

Kordopatis, G., Recio-Blanco, A., de Laverny, P., et al. 2011, A\&A, 535, A107

Kordopatis, G., Gilmore, G., Steinmetz, M., et al. 2013, AJ, 146, 134

Kordopatis, G., Wyse, R. F. G., Gilmore, G., et al. 2015, A\&A, 582, A122

Krtička, J., Kubát, J., \& Krtičková, I. 2020, A\&A, 635, A173

Lallement, R., Capitanio, L., Ruiz-Dern, L., et al. 2018, A\&A, 616, A132

Lightkurve Collaboration (Cardoso, J. V. d. M. a., et al.) 2018, Lightkurve: Kepler and TESS time series analysis in Python

Löbling, L., Maney, M. A., Rauch, T., et al. 2019, MNRAS, 441, 2831

Luo, A. L., Zhao, Y. H., Zhao, G., et al. 2016, VizieR Online Data Catalog: V/149

Luo, A. L., Zhao, Y. H., Zhao, G., et al. 2019, VizieR Online Data Catalog: $\mathrm{V} / 164$

Majewski, S. R., Schiavon, R. P., Frinchaboy, P. M., et al. 2017, AJ, 154, 94

Malasan, H. L., Yamasaki, A., \& Kondo, M. 1991, AJ, 101, 2131

Marsh, T. R. 1989, PASP, 101, 1032

Maxted, P. F. L., Heber, U., Marsh, T. R., \& North, R. C. 2001, MNRAS, 326, 1391

Miller Bertolami, M. M. 2016, A\&A, 588, A25

Mitrofanova, A. A., Shimansky, V. V., Borisov, N. V., Spiridonova, O. I., \& Gabdeev, M. M. 2016, Astron. Rep., 60, 252

Moe, M., Kratter, K. M., \& Badenes, C. 2019, ApJ, 875, 61

Nagel, T., Schuh, S., Kusterer, D. J., et al. 2006, A\&A, 448, L25

Napiwotzki, R., Christlieb, N., Drechsel, H., et al. 2001, Astron. Nachr., 322, 411

Nebot Gómez-Morán, A., Gänsicke, B. T., Schreiber, M. R., et al. 2011, A\&A 536, A43

Ness, M., Hogg, D. W., Rix, H. W., Ho, A. Y. Q., \& Zasowski, G. 2015, ApJ, 808,16

Nie, J. D., Wood, P. R., \& Nicholls, C. P. 2012, MNRAS, 423, 2764

Parsons, S. G., Rebassa-Mansergas, A., Schreiber, M. R., et al. 2016, MNRAS, 463, 2125

Pastetter, L., \& Ritter, H. 1989, A\&A, 214, 186

Peterson, R. C., Tarbell, T. D., \& Carney, B. W. 1983, ApJ, 265, 972

Pietrinferni, A., Cassisi, S., Salaris, M., \& Castelli, F. 2004, ApJ, 612, 168

Press, W. H., Teukolsky, S. A., Vetterling, W. T., \& Flannery, B. P. 1992, Numerical Recipes in C. The Art of Scientific Computing (Cambridge: Cambridge University Press)

Rauch, T., Ziegler, M., Werner, K., et al. 2007, A\&A, 470, 317

Rebassa-Mansergas, A., Ren, J. J., Parsons, S. G., et al. 2016, MNRAS, 458 3808

Rebassa-Mansergas, A., Ren, J. J., Irawati, P., et al. 2017, MNRAS, 472 4193

Rebassa-Mansergas, A., Toonen, S., Korol, V., \& Torres, S. 2019, MNRAS, 482 3656

Recio-Blanco, A., de Laverny, P., Kordopatis, G., et al. 2014, A\&A, 567, A5

Reindl, N., Geier, S., Kupfer, T., et al. 2016, A\&A, 587, A101
Reindl, N., Schaffenroth, V., Miller Bertolami, M. M., et al. 2020, A\&A, 638, A93

Ritter, H. 1986, A\&A, 168, 105

Santander-García, M., Rodríguez-Gil, P., Corradi, R. L. M., et al. 2015, Nature, 519,63

Schlafly, E. F., \& Finkbeiner, D. P. 2011, ApJ, 737, 103

Schlegel, D. J., Finkbeiner, D. P., \& Davis, M. 1998, ApJ, 500, 525

Schreiber, M. R., Gänsicke, B. T., Rebassa-Mansergas, A., et al. 2010, A\&A, 513, L7

Schuh, S. L., Dreizler, S., Deetjen, J. L., \& Göhler, E. 2003, Balt. Astron., 12, 167

Shimansky, V. V., Borisov, N. V., Nurtdinova, D. N., et al. 2015, Astron. Rep., 59,199

Silvestri, N. M., Hawley, S. L., \& Oswalt, T. D. 2005, AJ, 129, 2428

Simon, T., \& Drake, S. A. 1989, ApJ, 346, 303

Sing, D. K., Holberg, J. B., Burleigh, M. R., et al. 2004, AJ, 127, 2936

Stelzer, B., Marino, A., Micela, G., López-Santiago, J., \& Liefke, C. 2013, MNRAS, 431, 2063

Stelzer, B., Damasso, M., Scholz, A., \& Matt, S. P. 2016, MNRAS, 463, 1844

Strassmeier, K. G. 2009, A\&ARv, 17, 251

Tian, H.-J., Liu, C., Carlin, J. L., et al. 2015, ApJ, 809, 145

Tian, H.-J., El-Badry, K., Rix, H.-W., \& Gould, A. 2020, ApJS, 246, 4

Tonry, J. L., Denneau, L., Heinze, A. N., et al. 2018, PASP, 130, 064505

Unglaub, K., \& Bues, I. 2000, A\&A, 359, 1042

Van der Swaelmen, M., Boffin, H. M. J., Jorissen, A., \& Van Eck, S. 2017, A\&A, 597, A68

Van Winckel, H. 2018, ArXiv e-prints, [arXiv:1809.00871]

Werner, K., \& Dreizler, S. 1999, J. Comput. Appl. Math., 109, 65

Werner, K., \& Herwig, F. 2006, PASP, 118, 183

Werner, K., Deetjen, J. L., Dreizler, S., et al. 2003, ASP Conf. Ser., 288, 31

Werner, K., Dreizler, S., \& Rauch, T. 2012, Astrophysics Source Code Library [record ascl:1212.015]

Werner, K., Rauch, T., \& Kruk, J. W. 2015, A\&A, 582, A94

Werner, K., Rauch, T., \& Kruk, J. W. 2017, A\&A, 601, A8

Werner, K., Rauch, T., \& Kruk, J. W. 2018a, A\&A, 609, A107

Werner, K., Rauch, T., \& Kruk, J. W. 2018b, A\&A, 616, A73

Werner, K., Rauch, T., \& Reindl, N. 2019, MNRAS, 483, 5291

Willems, B., \& Kolb, U. 2004, A\&A, 419, 1057

Wilson, O. C. 1963, ApJ, 138, 832

Wilson, O. C. 1968, ApJ, 153, 221

Wright, E. L., Eisenhardt, P. R. M., Mainzer, A. K., et al. 2010, AJ, 140, 1868

Zechmeister, M., \& Kürster, M. 2009, A\&A, 496, 577

Zhao, J. K., Oswalt, T. D., Rudkin, M., Zhao, G., \& Chen, Y. Q. 2011, AJ, 141, 107

Zhao, G., Zhao, Y.-H., Chu, Y.-Q., Jing, Y.-P., \& Deng, L.-C. 2012a, Res. A\&A, 12,723

Zhao, J. K., Oswalt, T. D., Willson, L. A., Wang, Q., \& Zhao, G. 2012b, ApJ, 746,144

Ziegler, M., Rauch, T., Werner, K., Köppen, J., \& Kruk, J. W. 2012, A\&A, 548, A109

Zorotovic, M., Schreiber, M. R., Gänsicke, B. T., et al. 2011, A\&A, 536, L3 